\title{
Forecasting Economic Processes
}

\author{
Michael P. Clements \\ Department of Economics, \\ University of Warwick
}

and

\author{
David F. Hendry* \\ Nuffield College, \\ Oxford.
}

March 30, 2008

\begin{abstract}
When the assumption of constant parameters fails, the in-sample fit of a model may be a poor guide to ex-ante forecast performance. We exposit a number of models, methods, and procedures that illustrate the impacts of structural breaks on forecast accuracy, and evaluate ways of improving forecast performance. We argue that a theory of economic forecasting which allows for model mis-specification and structural breaks is feasible, and may provide a useful basis for interpreting and circumventing systematic forecast failure in macroeconomics. The empirical time series of consumers' expenditure, and Monte Carlo simulations, illustrate the analysis.

Keywords. Forecasting, structural breaks, intercept corrections, consumers' expenditure, Monte Carlo .
\end{abstract}

\section{Introduction}

This paper illustrates some of our recent results on macroeconomic forecasting in the presence of structural breaks. The approach departs in several ways from the traditional theory of economic forecasting (see e.g., Klein, 1971), and suggests a need to reappraise practices and modelling approaches which appear redundant in the classical paradigm. If the data generating process truly was stationary (perhaps after differencing or cointegration transforms) with time-invariant parameters, and if we were equipped with a forecasting model which coincided with that process, then it would be hard to justify some commonplace macro-econometric forecasting practices, such as intercept corrections (or residual adjustments), or to explain some of the predictive failures (or systematic forecast errors) that have occurred in recent economic forecasting.

It is widely recognised that published forecasts are rarely purely model-based, and adjustments are often made to arrive at a final forecast (see, e.g., Turner, 1990, for case studies of the impacts of such adjustments on the final forecasts). Thus, published economic forecasts reflect in varying degrees the properties of the models and the skills of the models' proprietors. Moreover, forecasters' adjustments do appear to improve forecast accuracy: see, for example, the work of the ESRC Macroeconomic Modelling Bureau, namely Wallis, Andrews, Fisher, Longbottom and Whitley (1986), especially Table 4.8; Wallis, Fisher, Longbottom, Turner and Whitley (1987), Figs. 4.3 and 4.4; and Wallis and Whitley (1991).

Nevertheless, as noted in Clements and Hendry (1995), confidence in macro-economic forecasting has periodically been punctured by episodes of dramatic predictive failure. Recent examples include the poor performance in predicting the consumer boom in the late 1980s, and the depth and duration of

${ }^{*}$ Financial support from the UK Economic and Social Research Council under grant L116251015 is gratefully acknowledged by both authors. 
the recession in the 1990s, with noteworthy antecedents including the under-prediction of post-war consumption, and the 1974-5 and 1979-81 recessions. Wallis (1989) discusses in more detail the forecasting record of the major UK model-based forecasting teams for the 1974-5 and 1979-81 recessions.

An econometric theory of economic forecasting will only deliver relevant conclusions about empirical forecasting if it adequately captures the appropriate aspects of the real world to be forecast. That a theory which posits a cointegrated-stationary, time-invariant data generating process (DGP), perfectly replicated by a forecasting model, is not consonant with an empirical track record of large predictive failures is unsurprising. However, the latter may be consistent with a theory that eschews these restrictive assumptions.

There are several related lines of research. First, Robert Fildes and others have noted anomalies between the outcomes of empirical-accuracy studies of univariate time-series forecasting methods, and a statistical paradigm of theoretical time-series analysis (see, for example, Box and Jenkins, 1976, and compare Fildes and Makridakis, 1995). Essentially, the forecasting methods that appear to work empirically in the forecasting competitions are not those which would have been predicted by statistical theory, and Fildes and Makridakis (1995) suggest that the most serious culprit is the assumption of constancy which underpins that paradigm: this matches the importance we attribute to structural breaks (see, for example, Clements and Hendry, 1996b, inter alia).

Secondly, the 'dynamic linear models' of West and Harrison (1989) (see also Pole, West and Harrison, 1994, for a wealth of applications) put parameter non-constancy centre stage. The difference does not lie in their allowing the parameters of the observation (measurement) equation to evolve according to a system (transition) equation, since the latter becomes the constant-parameter basis. However, these authors also stress the importance of interventions based on an ongoing monitoring of forecast performance, and the adequacy of the model is questioned when the latest observations are in a tail of the forecast distribution, possibly leading to a subjective intervention.

Our own research focuses on systems of cointegrated relations representing macro-econometric models, when economies are subject to unanticipated, large regime shifts, as when the UK left the ERM in September 1992. The paper illustrates, by empirical examples and Monte Carlo simulations, a number of the models, methods, and procedures that we have analyzed more rigorously elsewhere, to investigate the quantitative impact of structural breaks on systematic forecast errors. We also show that:

'some methods are more appropriate and/or robust than others in dealing with different types of ... possible changes': Fildes and Makridakis, 1995, p. 302.

We use as a framework one of the simplest DGPs exhibiting a structural break, namely a first-order scalar autoregression. The scalar framework is for expositional simplicity only: in the context of economic forecasting, a multivariate approach that captures behavioural relationships between variables is usually essential (see, for example, Hendry and Doornik, 1994), especially since economic policy frequently responds to forecast changes. Nevertheless, we think it is useful to demonstrate that most of the implications from the associated theory are consistent with the results for scalar processes. The analytical simplicity also brings to the fore the conceptual issues involved. In each case, the reader is referred to literature where a more detailed treatment is available.

The DGP is a simple member of the class of models recently considered by Andrews (1993), who develops 'one-shot' tests of structural change which are designed to test for a single break in a time series. More generally, the sequential testing procedure of Chu, Stinchcombe and White (1996) 'monitors' for structural change as new observations accrue, so is potentially relevant in the forecasting context, and Chu et al. (1996) show that 'one-shot' tests cannot be repeatedly applied as new observations arrive (the size of that procedure would go to one). We shall not be concerned with the appropriate distribu- 
tional theory for testing such hypotheses, as the cases of concern here are when forecast errors are so large that no test is necessary to discern if a change has occurred.

We consider approximating a process with a break by a variety of types of 'model', ranging from predicting the sample mean of the process, to 'no change' type forecasts, and include members of the autoregressive, integrated moving-average (ARIMA) class of the Box and Jenkins (1976) time-series modelling tradition. The estimators considered include least squares and instrumental variables using 1-step and multi-step (or 'adaptive forecasting') criteria; and the forecasting procedures include unmodified models as well as intercept corrections. The choice of evaluation criterion is also considered.

The plan of the paper is as follows. In section 2, we describe the DGP. This is an autoregressive process with a one-off change in the mean only, at an exogenously determined point of time. Section 3 describes the forecasting methods used to analyse this DGP, and section 4 introduces the empirical example which illustrates the performance of the methods on an actual data series. Sections 5 to 11 present analytical results for the various forecasting methods, as well as the results for the artificial (simulated) data and the actual empirical data, demonstrating in most cases an acceptable concordance. Finally, section 12 concludes and summarises.

\section{The data generation process}

The DGP is given by the following scalar first-order autoregressive process:

$$
y_{t}=\rho y_{t-1}+\mu^{*}+\epsilon_{t}
$$

where, for most of the discussion, we set $\rho=0$, and assume that $\epsilon_{t} \sim \operatorname{IN}\left(0, \sigma_{\epsilon}^{2}\right)$, denoting an independent normal distribution with zero mean and constant variance $\sigma_{\epsilon}^{2}$. We allow $\mu^{*}$ to take on two values: $\mu_{1}$ when $t \leq \tau$, and $\mu_{2}$ when $t>\tau$. Thus, the baseline DGP is simply white noise $(\rho=0)$ with a shift in mean at time $t=\tau+1$. Letting $y_{t}=\Delta x_{t}$ where $x_{t}=\log X_{t}$ is a natural interpretation, so that $\mu^{*}$ is the growth rate, and the underlying levels process $X_{t}$ is integrated (after a log transform). Vector generalizations are presented in the appendix when these add insight. When we consider the small-sample properties of estimators and moments of the forecast-error distributions, we shall use Monte Carlo, and set $\mu_{1}=1, \mu_{2}=10, \tau=\frac{T}{2}, T=100, \sigma_{\epsilon}^{2}=1$, replicating the process 10,000 times.

\section{Forecasting methods}

We are primarily concerned with short-term forecasts of $y_{t}$, so consider horizons $h$ of 1 to 4 -steps ahead, where the forecast origin is taken to be $T$. The process in (1) is invariant to specifying the dependent variable as $y_{t}$ or $\Delta y_{t}$ so long as $y_{t-1}$ enters unrestrictedly. But, beyond 1-step, most conventional evaluation criteria are not, and it matters for which transform of the dependent variable forecast errors are evaluated (see Clements and Hendry, 1993).

The models of $y_{t}$ we consider are summarized in table 1, and we take each in turn in subsequent sections. Section 11 allows for $\rho \neq 0$, in which case, following the impact of the break at period $\tau$, the process will undergo a period of adjustment to the new equilibrium, in contrast to instantaneous adjustment when $\rho=0$. The results of the Monte Carlo for the models/methods described in table 1 are presented in table 2, which reports the mean squared forecast errors (MSFEs), the squared forecast biases, and the forecast error variances, for horizons $1-4$. The Monte Carlo distributions of the parameter estimates of the various models are shown in table 4 . We refer to these as required below. 
Table 1 Forecasting Models.

\begin{tabular}{|c|c|c|c|}
\hline Label & ARIMA description & Constant & Comment \\
\hline $\mathrm{M}_{1}$ & - & constant & Sample mean predictor \\
$\mathrm{M}_{2}$ & ARIMA $(1,0,0)$ & yes & 1-step minimization \\
$\mathrm{M}_{3}$ & ARIMA $(1,0,0)$ & yes & Dynamic estimation \\
$\mathrm{M}_{4}$ & ARIMA $(0,1,0)$ & yes & Unit root imposed \\
$\mathrm{M}_{5}$ & ARIMA $(0,1,0)$ & no & Unit root imposed \\
$\mathrm{M}_{6}$ & ARIMA $(0,1,1)$ & yes & IMA \\
$\mathrm{M}_{7}$ & ARIMA $(0,1,1)$ & no & IMA \\
$\mathrm{M}_{8}$ & ARIMA $(1,0,1)$ & yes & ARMA \\
\hline
\end{tabular}

\section{An empirical example}

For our empirical illustration, we use real consumers' expenditure on non-durables and services in the United Kingdom (denoted $C_{t}$ ), quarterly (not seasonally adjusted) from 1961(1) to 1992(4). There is a long history of predictive failure of this variable using econometric models, as witnessed by the results in Hendry (1974), Davidson, Hendry, Srba and Yeo (1978), Muellbauer and Murphy (1989), Carruth and Henley (1990), and Hendry (1994) inter alia. Figure 1a-d shows the time series of the level (denoted $\left.r c n d=C_{t}\right)$, the log level $\left(\right.$ Lrcnd $\left.=c_{t}\right)$, the first difference $\left(\right.$ L Lrcnd $\left.=\Delta c_{t}=c_{t}-c_{t-1}\right)$, and the fourth difference or annual growth (D4Lrcnd $\left.=\Delta_{4} c_{t}=c_{t}-c_{t-4}\right){ }^{1}$
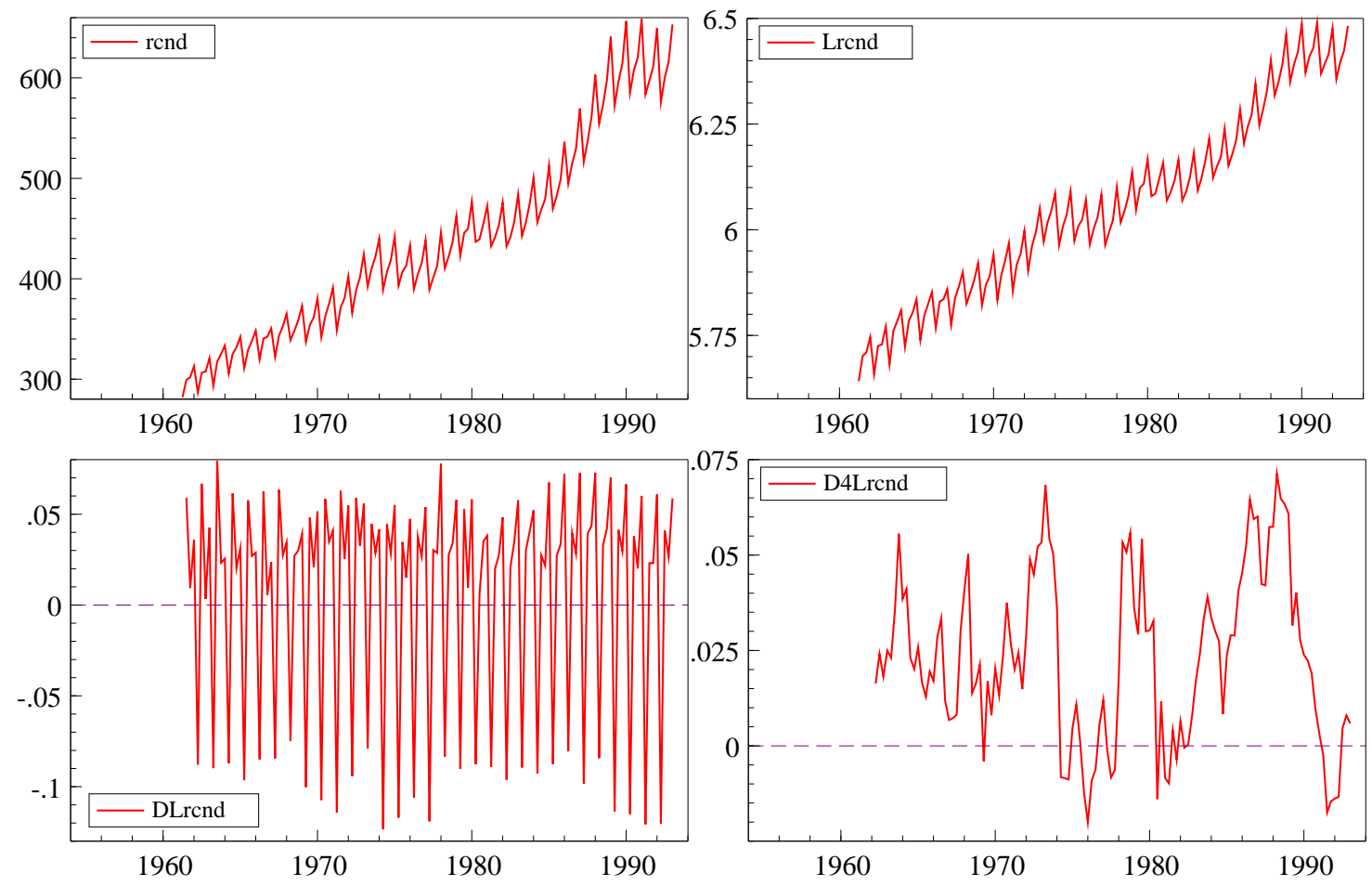

Figure 1 Time series of real consumers' expenditure in the UK.

\footnotetext{
${ }^{1}$ The four panels are numbered from left to right and top to bottom. All empirical estimates and graphs are based on GiveWin and PcGive for Windows: see Hendry and Doornik (1996a) and Hendry and Doornik (1996b). The simulations were performed using the Gauss programming language, Aptech Systems, Inc., Washington.
} 
The original series is visually highly seasonal, around a strong trend; the log transform is variance stabilizing so is used below; the quarterly growth series is dominated by the movements between seasons, which are of the order of $15 \%$ switches and may reflect seasonal unit roots. The annual growth has usually been positive, with only a few falls. We focus on $\Delta_{4} c_{t}$ as the variable $y_{t}$ (for $\rho \neq 0$ ) in (1), and presume that a possible change in the underlying growth rate occurred around 1980. Over the whole sample, the mean and standard deviation of $\Delta_{4} c_{t}$ are almost equal at $2.3 \%$ and $2.2 \%$ respectively.

To show the nature of the predictive failure, we consider 1-step ex post forecasts for the equation in Davidson et al. (1978) (known as DHSY), which is close to the equation in the Treasury model during the early 1980s. Letting $i_{t}$ denote real personal disposable income and $p_{t}$ its implicit deflator, the equation relates the annual changes with an equilibrium-correction mechanism (EqCM) from the previous year's differential between consumption and income such that, on a steady-state growth path for income, consumption is proportional to income. The estimates over the sample 1962(2)-1982(4) are: ${ }^{2}$

$$
\begin{aligned}
\widehat{\Delta_{4} c_{t}} & =\underset{(0.04)}{0.37} \Delta_{4} i_{t}+\underset{(0.04)}{0.15} \Delta_{4} i_{t-1}-\underset{(0.02)}{0.11}(c-i)_{t-4}-\underset{(0.02)}{0.13} \Delta_{4} p_{t} \\
\mathrm{R}^{2} & =0.89 \widehat{\sigma}_{e}=0.96 \% \mathrm{~F}_{\mathrm{ar}}(5,74)=0.96 \mathrm{~F}_{\mathrm{arch}}(4,71)=1.19 \\
\chi_{\text {nd }}^{2}(2) & =3.23 \mathrm{~F}_{\text {het }}(8,70)=1.29 \mathrm{~F}_{\mathrm{re}}(1,78)=3.67 \mathrm{~J}=0.55 \mathrm{SC}=-9.12
\end{aligned}
$$

$\mathrm{R}^{2}$ is the squared multiple correlation coefficient, $\widehat{\sigma}_{e}$ is the residual standard deviation expressed as a percentage of $C_{t}, \mathrm{SC}$ is the Schwarz criterion; and the diagnostic tests are of the form $\mathrm{F}_{\mathrm{j}}(k, T-l)$ which denotes an F-test against the alternative hypothesis $\mathrm{j}$ for: $5^{\text {th }}$-order residual serial correlation $\left(F_{a r}\right.$ : see Godfrey, 1978), $4^{t h}$-order residual autoregressive conditional heteroscedasticity $\left(F_{\text {arch }}\right.$ : see Engle, 1982), heteroscedasticity ( $F_{\text {het }}$ : see White, 1980); the RESET test ( $F_{\text {re }}$ : see Ramsey, 1969); the joint parameter constancy tests in Hansen (1992) $(J)$, and a chi-square test for normality $\left(\chi_{\text {nd }}^{2}(2)\right.$ : see Doornik and Hansen, 1994): ${ }^{*}$ and ${ }^{* *}$ denote significance at the 5\% and $1 \%$ levels respectively. All the in-sample tests are acceptable, but Figure $2 \mathrm{a}-\mathrm{d}$ shows the fitted, actuals and forecast values, their cross plot with separate regression lines pre and post 1982; the residuals and forecast errors scaled by the equation standard error; and the forecasts with 1-step 95\% confidence bands around the forecasts. The forecast and sample regressions have distinctly different slopes and the post-sample residuals greatly exceed the in-sample ones. Many forecasts lie outside their one-off confidence intervals, and the Chow (1964) constancy test over 1983(1)-1992(3) yields $F(40,79)=2.90^{* *}$ which rejects at the $1 \%$ level, consistent with the low correlation between outturns and forecasts over the later period in $2 \mathrm{a}$.

Considerable effort has been devoted to understanding the causes of this predictive failure, and there are many potential explanations ranging from inaccurate data, inappropriate economic analysis, an invalid model class, bad methodology, structural change (particularly the financial deregulation of the mid-1980s and possibly demographic change), and omitted variables (mainly wealth related measures): see the excellent review in Muellbauer (1994). The main point of the above illustration is to demonstrate that a change of some form did occur, and that previously-successful equations did not forecast through that change; partial statistical explanations will emerge as we proceed. Hendry and Doornik (1997) present theoretical analyses of several of the other putative causes, and demonstrate the central role of deterministic shifts.

\footnotetext{
${ }^{2}$ These differ from the original estimates mainly due to problems with the data revisions implemented in the early 1990 s for the mid-1970s data: see Hendry (1994) for a discussion. Davidson et al. (1978) called the level feedback an error-correction mechanism, but as will be seen, this is a misnomer.
} 

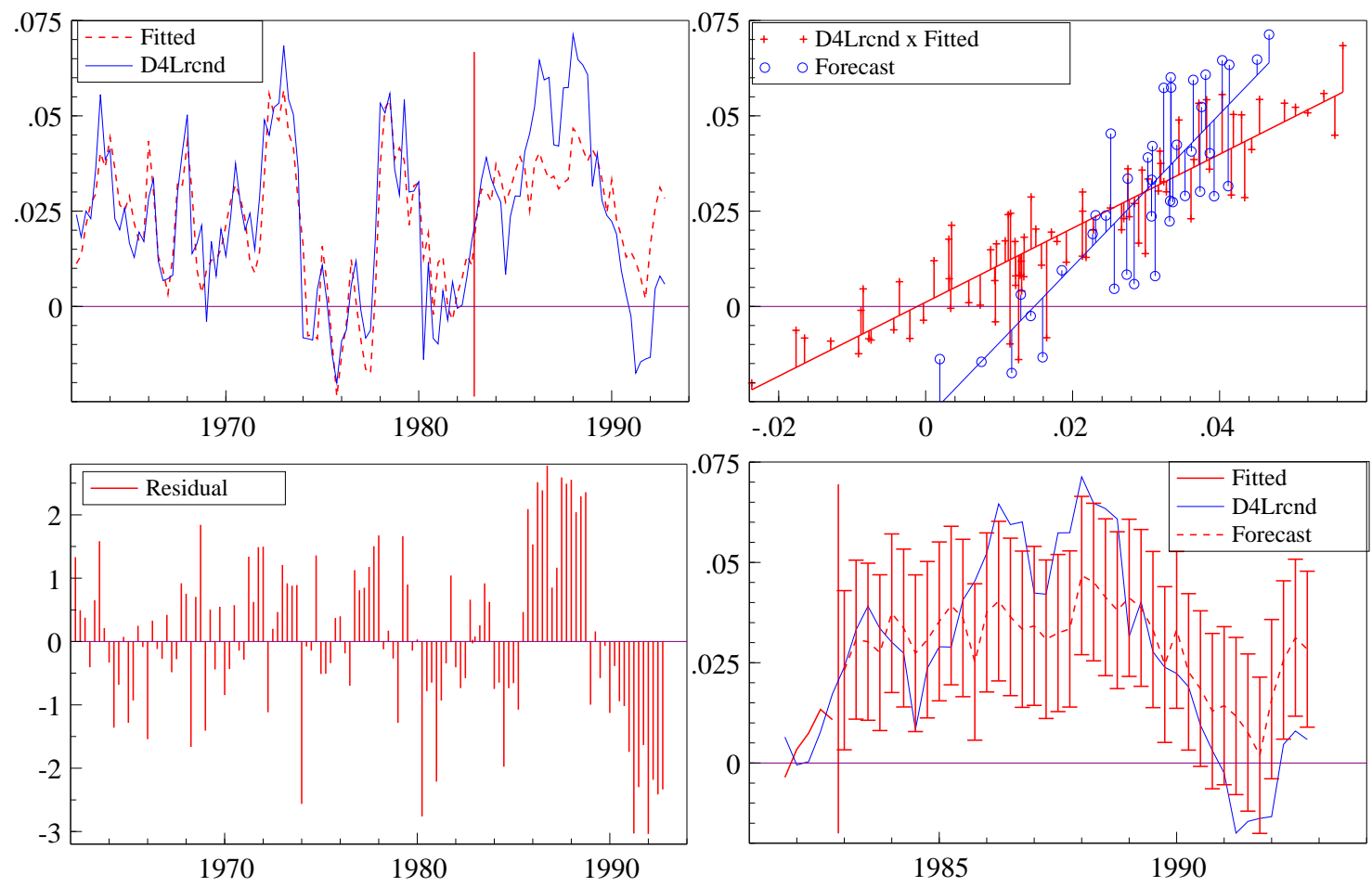

Figure 2 Graphical statistics for the DHSY model.

\section{The sample mean as a predictor}

The forecast function for $\mathbf{M}_{1}$ is simply the sample mean. For $\rho=0$ and $\tau=\frac{T}{2}$ the population value of the sample mean is $\mu_{p}=\frac{1}{2}\left(\mu_{1}+\mu_{2}\right)$. The form of this predictor is that forecasts are not conditional on the value(s) of the process around the forecast origin, and forecasts are badly biased when the recent behaviour of the process is very different from the history on which $\mu_{p}$ is based. Ignoring the imprecision in estimating $\mu$, the forecast error variance is only $\sigma_{\epsilon}^{2}$, the minimum obtainable using $y_{T+h}=\mu_{2}$. But on MSFE comparisons (the sum of the squared bias and forecast-error variance), this strategy fares poorly:

$$
\text { bias }=\mathrm{E}\left[y_{T+h}-\mu_{p}\right]=\mu_{2}-\frac{1}{2}\left(\mu_{1}+\mu_{2}\right)=\frac{\delta_{\mu}}{2}
$$

where $\delta_{\mu}=\mu_{2}-\mu_{1}$ can be made arbitrarily large relative to $\sigma_{\epsilon}$. When $\rho \neq 0$, the bias is scaled up by $(1-\rho)^{-1}$ and the forecast-error standard deviation by $1 / \sqrt{ }\left(1-\rho^{2}\right)$, since:

$$
y_{t}=\frac{\mu^{*}}{(1-\rho)}+u_{t} \text { where } u_{t}=\rho u_{t-1}+\epsilon_{t}
$$

so that $\sigma_{u}^{2}=\sigma_{\epsilon}^{2} /\left(1-\rho^{2}\right)$, yielding:

$$
\operatorname{MSFE}_{1}(y: h)=\frac{\sigma_{\epsilon}^{2}}{\left(1-\rho^{2}\right)}+\frac{\delta_{\mu}^{2}}{4(1-\rho)^{2}} .
$$

\section{Differencing}

As a polar case, consider $\mathrm{M}_{5}$. Let $y_{j \mid i}$ denote the forecast function for the model under consideration, where $i$ is the forecast origin (on which the forecast is conditioned), and $j$ is the period being forecast. 
Here $y_{T+h \mid T}=y_{T}$, so that the history of the process, other than the value at the forecast origin, is irrelevant. 'Complete' conditioning on the origin would appear to be a good idea when the future is exactly like the present, but will be costly if the present is atypical, and if in the future the process returns to its long-run average. Hence, differencing offers potential advantages for short-term forecasting when the present pattern persists for a number of quarters, but may yield unreliable forecasts over longer horizons. As an extreme example, suppose the shift in mean to $\mu_{2}$ at period $\tau+1$ is reversed in period $\tau+2$. Multi-step forecasts conditional on period $\tau+1$ will fare badly compared to using the sample mean. The sample mean is robust to irregular or outlier observations at the forecast origin, whereas differencing quickly incorporates change, and gains if that change persists. These are extremely simple examples of forecasting methods but serve to illustrate that when constancy fails to hold, quite different approaches to forecasting may be called for, depending upon the expected nature of the non-constancy. Fildes and Makridakis (1995), p.302, remark upon more elaborate models, and changes in trend rather than mean, but the upshot of their argument is similar. ${ }^{3}$

While differencing may reduce bias, since the process is stochastic, it will not repeat the previous period (barring an event with probability zero), which has implications for the forecast error variance attached to this type of predictor. Since the structural break (mean-shift) has occurred prior to the forecast origin (at period $\frac{T}{2}$ compared to period $T$ ), differencing results in largely unbiased forecasts when the constant is not estimated. This is because:

$$
\mathrm{E}\left[y_{T+h \mid T}\right]=\mathrm{E}\left[y_{T}\right]=\mu^{*},
$$

and:

$$
\mathrm{E}\left[y_{T+h}\right]=\mu^{*} .
$$

However, the cost in forecast error variance arises because the predictor projects $y_{T}$, which is $\mu_{2}+\epsilon_{T}$, so there is an 'error' in the present. Because the future value of the process is $\mu_{2}+\epsilon_{T+h}$, and since $\epsilon_{T}$ and $\epsilon_{T+h}$ are independent for all $h$, the forecast error variance is twice the minimum. Formally, when $\rho=0$ the unconditional variance component is:

$$
\mathrm{E}\left[\left(\mu^{*}+\epsilon_{T+h}-y_{T}\right)^{2}\right]=\sigma_{\epsilon}^{2}+\left(\mu^{*}\right)^{2}+\mathrm{E}\left[y_{T}^{2}\right]-2 \mu^{*} \mathrm{E}\left[y_{T}\right]=2 \sigma_{\epsilon}^{2} .
$$

When a constant is included $\left(\mathrm{M}_{4}\right)$, then ignoring parameter estimation uncertainty, the forecast function is:

$$
y_{T+h \mid T}=y_{T}+h T^{-1} \delta_{\mu}
$$

with forecast bias:

$$
\text { bias }=\mathrm{E}\left[y_{T+h}-y_{T+h \mid T}\right]=-h T^{-1} \delta_{\mu},
$$

so that the bias is small but increases with the forecast horizon. Again, in large samples, the forecast error variance is $2 \sigma_{\epsilon}^{2}$ (ignoring the impact of estimating the constant term).

The empirical illustration of these two cases is a revealing confirmation of the analysis: differencing corresponds to estimating:

$$
\Delta_{4} c_{t}=\alpha+\Delta_{4} c_{t-1}+u_{t}
$$

where $\alpha=0$ for $\mathrm{M}_{5}{ }^{4}$ Since $\widehat{\alpha}$ is tiny $\left(10^{-5}\right)$, we report only the results for $\alpha=0$ where the 1-step forecasts are shown in Figure 3a-d. There is no evidence of predictive failure, the two regressions (in

\footnotetext{
${ }^{3}$ For example, they suggest that single exponential smoothing or damped trend smoothing may be more robust to a range of changes in trend than ARIMA models.

${ }^{4}$ We fit this by non-linear least squares to maintain $\Delta_{4} c_{t}$ as the dependent variable, imposing the coefficient of $\Delta_{4} c_{t-1}$ at unity.
} 
and out of sample) have nearly equal slopes and the post-sample residuals are smaller than the in-sample. Now $\widehat{\sigma}_{u}=1.47 \%$, so the residual variance exceeds that based on $\widehat{\sigma}_{e}$ by more than $100 \%$. However, there is evidence of residual autocorrelation at 4 lags, as $F_{\text {ar }}(5,74)=5.04$, and non-normality, as $\chi_{\text {nd }}^{2}(2)=6.57$. To the extent that the predictive failure in (2) is due to a shift in the equilibrium mean, differencing offsets that and allows nearly unbiased forecasts with doubled error variance, as anticipated from the analysis for $\mathrm{M}_{5}$.
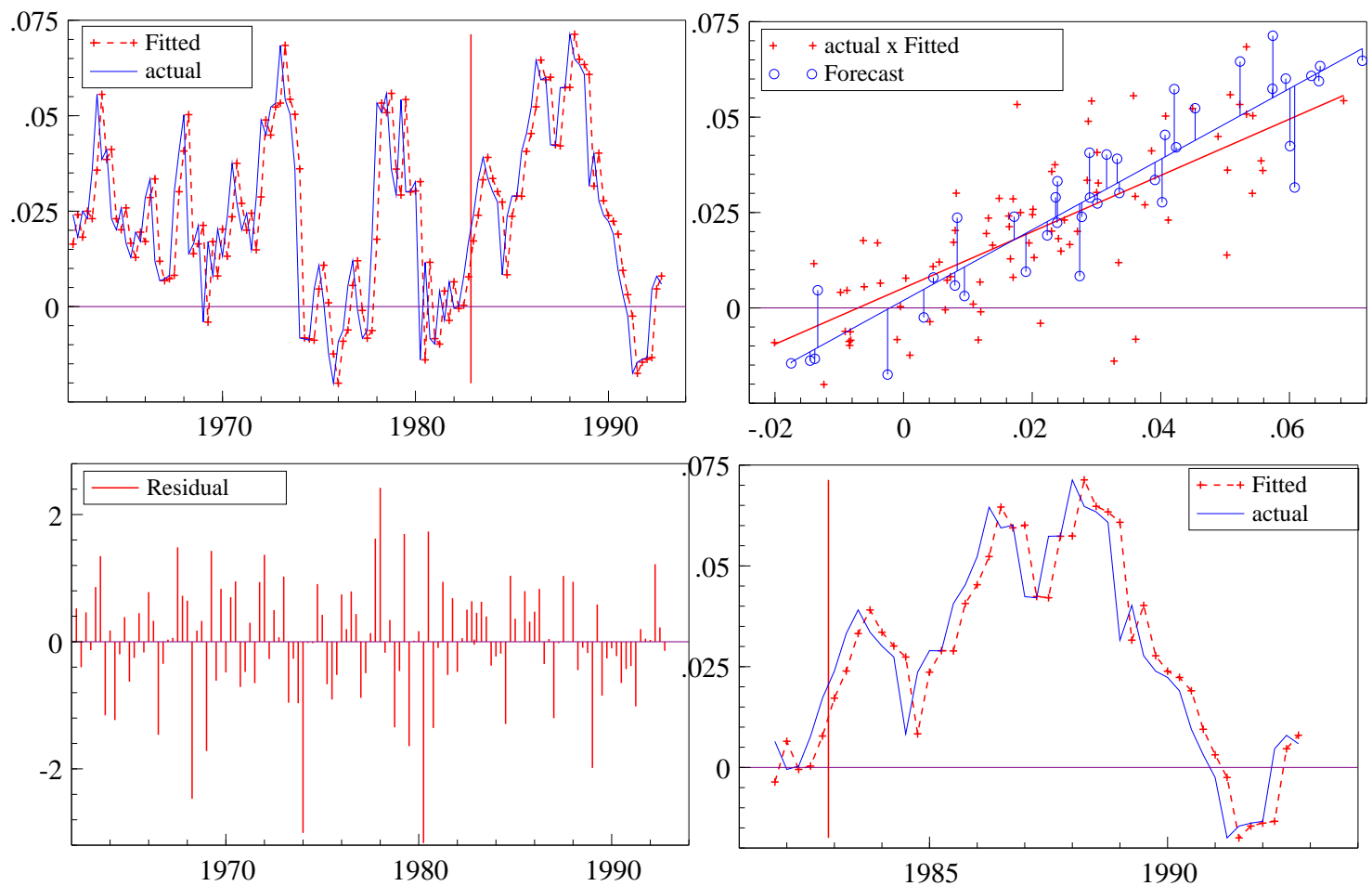

Figure 3 Graphical statistics for the differenced model.

\section{AR(1) model: 1-step estimation}

Consider now $\mathrm{M}_{2}$, the AR (1) with a constant term, estimated by OLS. Thus the model is:

$$
y_{t}=\alpha+\beta y_{t-1}+v_{t}
$$

and for the break outlined above, $0<\beta<1$ with $\mu_{1}<\frac{\alpha}{1-\beta}<\mu_{2}$. Also, $\beta \rightarrow 1$ as $\delta_{\mu} \rightarrow \infty$ where $\alpha$ and $\beta$ are the plims of the drift and slope parameters of an AR (1) model when the DGP is given by (1). The forecast function is (ignoring parameter estimation uncertainty):

$$
y_{T+h \mid T}=\alpha \sum_{i=0}^{h-1} \beta^{i}+\beta^{h} y_{T} \rightarrow \frac{\alpha}{1-\beta} \text { as } h \rightarrow \infty .
$$

Let $\mu^{+}=\frac{\alpha}{1-\beta}$. Then $\mu^{+}$is the long-run mean of the process from the historical data, which incorporates the two regimes. The long-run mean of the process after $t=\tau$ is $\mu_{2}$, and $\mu^{+}<\mu_{2}$, so the forecasts are biased for large $h$. Incorporating historical information on the long-run mean of the process leads to biased predictions as the earlier information is outdated. 
Write (4) as:

$$
\Delta y_{t}=(\beta-1)\left(y_{t-1}-\frac{\alpha}{1-\beta}\right)+v_{t}
$$

and consider forecasting without the term $\left(y_{t-1}-\mu^{+}\right)$: this delivers the differenced model $\mathrm{M}_{5}$. Thus, the comparison between $\mathrm{M}_{2}$ and $\mathrm{M}_{5}$ is the scalar analogue of the comparison in Clements and Hendry (1996b) between the vector equilibrium-correction model (VEqCM) and the vector autoregression in the differences of the variables (DVAR), which neglects the long-run means of the cointegrated processes.

In terms of the scalar example, from the Monte Carlo, $\alpha, \beta$ are estimated as:

$$
\widehat{y}_{t}=\underset{[0.0034]}{0.452}+\underset{[0.0001]}{0.934 y_{t-1}}
$$

where the coefficients are the Monte Carlo estimates (averages over replications), and the figures in brackets are the standard deviations of the estimates across replications. Thus:

$$
\text { bias }=\mathrm{E}\left[y_{T+h}-y_{T+h \mid T}\right]=\mu_{2}-\mu^{+}=10-6.82=3.18 \text { for large } h .
$$

The Monte Carlo estimates of the (squared) biases for $h=1, \ldots, 4$, are shown in table 2, confirming that the forecasts are biased, while those from $\mathrm{M}_{5}$ are unbiased.

The forecast error variances (asymptotically, ignoring the contributions from parameter estimation) are given by:

$$
\begin{gathered}
\mathrm{E}\left[\left(y_{T+h}-y_{T+h \mid T}-\mathrm{E}\left[y_{T+h}-y_{T+h \mid T}\right]\right)^{2}\right]= \\
\mathrm{E}\left[\left\{\epsilon_{T+h}-\left(\alpha \sum_{i=0}^{h-1} \beta^{i}+\beta^{h} y_{T}-\mathrm{E}\left[\alpha \sum_{i=0}^{h-1} \beta^{i}+\beta^{h} y_{T}\right]\right)\right\}^{2}\right]= \\
\mathrm{E}\left[\left(\epsilon_{T+h}-\beta^{h} \epsilon_{T}\right)^{2}\right]=\sigma_{\epsilon}^{2}\left(1+\beta^{2 h}\right) .
\end{gathered}
$$

The forecast error variance is always less than from differencing (since $\beta<1$ ) and declines towards the minimum attainable as $h$ increases $\left(\sigma_{\epsilon}^{2}\right)$ : that this does not happen in the Monte Carlo is due to parameter estimation effects.

Empirically, for UK consumption we obtain:

$$
\begin{aligned}
& \widehat{\Delta_{4} c_{t}}=\underset{(0.0017)}{0.0052}+\underset{(0.07)}{0.74 \Delta_{4} c_{t-1}} \\
& \mathrm{R}^{2}=0.54 \widehat{\sigma}_{v}=1.38 \% \mathrm{~F}_{\mathrm{ar}}(4,77)=3.44^{* *} \mathrm{~F}_{\mathrm{arch}}(4,73)=0.40 \\
& \chi_{\text {nd }}^{2}(2)=5.60 \mathrm{~F}_{\text {het }}(2,78)=1.77 \mathrm{~F}_{\mathrm{re}}(1,80)=0.06 \mathrm{~J}=0.50 \quad \mathrm{SC}=-8.48
\end{aligned}
$$

The fit is little better than (3), as Figure 4a-d confirms, whereas the forecasts are poorer in terms of tracking, though never significantly bad.

\section{Intercept corrections}

Intercept corrections refer to the practice of specifying non-zero values for a model's error terms over the forecast period. Hendry and Clements (1994) provide a general theory of the role of intercept corrections in macro-econometric forecasting and Clements and Hendry (1996b, 1996a) focus on their role in offsetting regime shifts. If the model's (in-sample) error is an innovation on the information set, 

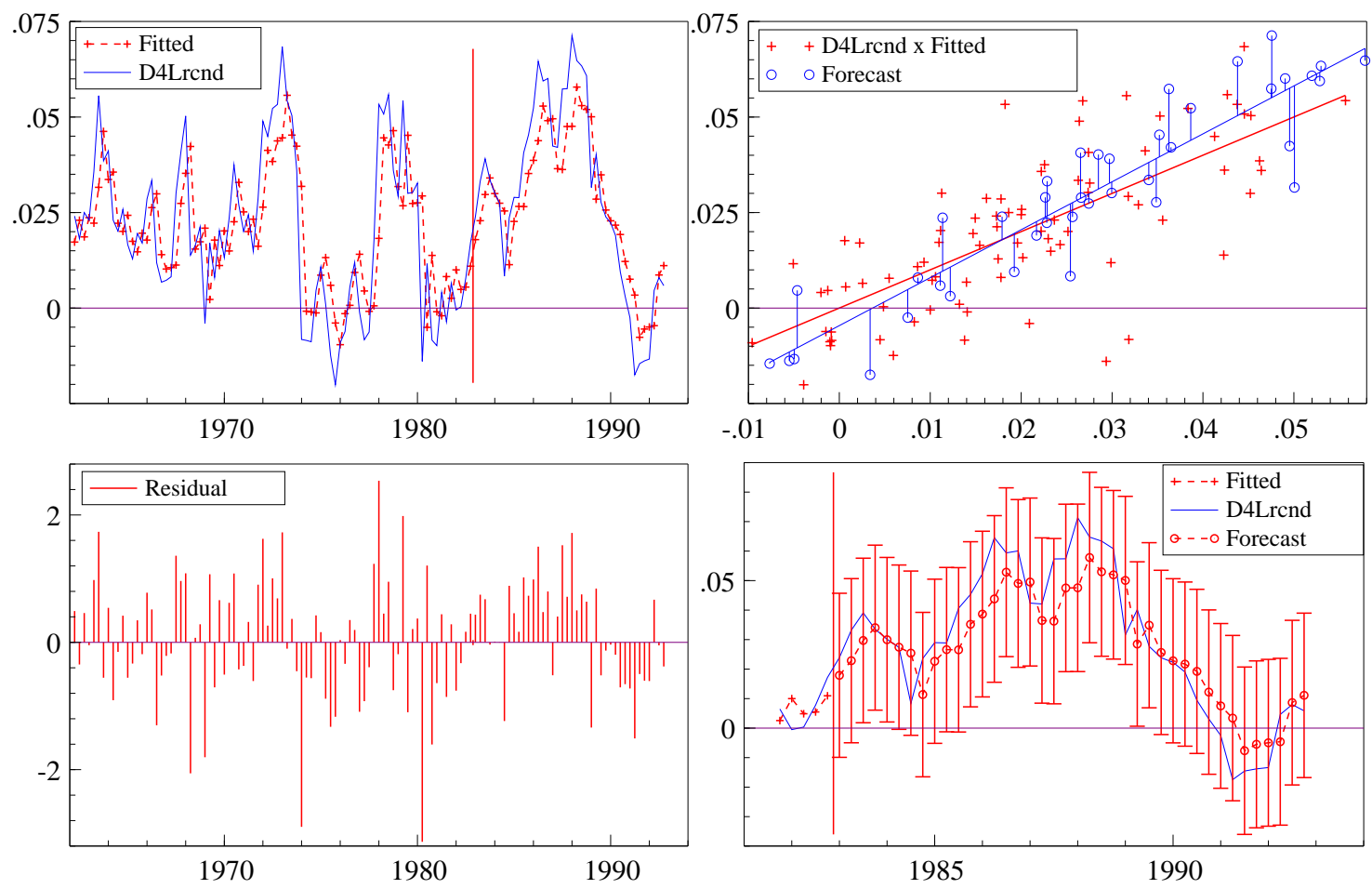

Figure 4 Graphical statistics for the AR (1) model, $\mathrm{M}_{2}$.

then in the absence of structural breaks over the future, or of other extraneous factors, it is natural to set the future values of the equations' error terms to zero.

Here, we consider the simplest form of intercept correction, whereby the forecaster reacts to perceived recent predictive failure by adding in the equation error in predicting $T$, the forecast origin. For $\mathrm{M}_{2}$, the AR (1) model, the period $T$ model error is, ignoring estimation uncertainty:

$$
\xi_{T}=y_{T}-y_{T \mid T-1}=\mu_{2}+\epsilon_{T}-\left(\alpha+\beta y_{T-1}\right)=\mu_{2}(1-\beta)-\alpha+(1-\beta L) \epsilon_{T},
$$

where $L$ is the lag operator, i.e., $L \epsilon_{t}=\epsilon_{t-1}$. When the adjustment is held constant over the forecast period, $\xi_{T}$ is added in at each step ahead:

$$
\bar{y}_{T+h \mid T}=\alpha+\beta \bar{y}_{T+h-1 \mid T}+\xi_{T}
$$

where $\bar{y}_{T \mid T}=y_{T}$, so that:

$$
\bar{y}_{T+h \mid T}=y_{T+h \mid T}+\xi_{T} \sum_{i=0}^{h-1} \beta^{i}=\left(\alpha+\xi_{T}\right) \sum_{i=0}^{h-1} \beta^{i}+\beta^{h} y_{T} .
$$

The bias from using (9) as a predictor is:

$$
\mathrm{E}\left[y_{T+h}-\bar{y}_{T+h \mid T}\right]=\mu_{2}-\frac{\alpha+\mathrm{E}\left[\xi_{T}\right]}{1-\beta}=0, \text { for large } h
$$

as $\mathrm{E}\left[\xi_{T}\right]=\mu_{2}(1-\beta)-\alpha$ from (7). When there is no structural break, $\mu_{2}=\mu_{1}$ and $\alpha=\mu_{2}, \beta=0$, so that $\mathrm{E}\left[\xi_{T}\right]=\mathrm{E}\left[\epsilon_{T}\right]=0$, and hence intercept correcting does not induce a bias. 
However, intercept corrections always result in an inflated forecast error variance. In our example, for large $h$ :

$$
\begin{aligned}
\mathrm{V}\left[y_{T+h}-\bar{y}_{T+h \mid T}\right] & =\mathrm{E}\left[\left(y_{T+h}-\bar{y}_{T+h \mid T}\right)^{2}\right] \\
& =\mathrm{E}\left[\left(\epsilon_{T+h}-\frac{\epsilon_{T}(1-\beta L)}{1-\beta}\right)^{2}\right]=\sigma_{\epsilon}^{2} \frac{2\left(1-\beta+\beta^{2}\right)}{(1-\beta)^{2}}
\end{aligned}
$$

constituting a doubling when there is no mean shift. Moreover, for $\beta$ close to unity, $\mathrm{V}[\cdot]$ gets very large. Intuitively, as $\beta \rightarrow 1$, the stochastic component of $\xi_{T}$, namely $\xi_{T}-\mathrm{E}\left[\xi_{T}\right] \rightarrow \Delta \epsilon_{T}$, and this is being multiplied by an only-just convergent sum $\sum_{i=0}^{h-1} \beta^{i}$. Evaluating (10) for 1-step forecasts gives:

$$
\mathrm{V}\left[y_{T+1}-\bar{y}_{T+1 \mid T}\right]=2\left(1+\beta+\beta^{2}\right)
$$

see the Monte Carlo estimates in table 2 where the error variance for $\mathrm{M}_{2}^{I C}$ (the intercept-corrected model $\mathrm{M}_{2}$ ) is 5.56 compared to 1.89 for $\mathrm{M}_{2}$, or 1.98 for $\mathrm{M}_{5}$, the differenced model.

In summary, in the absence of a structural break, the differenced model and intercept correcting (IC) strategies both yield unbiased forecasts and doubled forecast error variances (relative to $y_{T+h \mid T}=\mu_{2}$ ). However, these two forecasting methods are differentially susceptible to structural breaks. For the DGP we consider here, the IC strategy fares the worst in terms of forecast error variance, but this is not necessarily the case generally (see the empirical example in Clements and Hendry, 1996b).

For consumers' expenditure, intercept correcting the 1-step forecasts yields the outcomes shown in Figure 5: the in-sample behaviour is identical by construction, but the forecasts track the outcomes, albeit with a much larger variance. Of course, the more pertinent issue is the effect of intercept correcting on (2), and as Figure 6 shows, the effect is dramatic: unbiased forecasts do indeed result, again with considerable volatility due to incorporating the whole of the previous error as well as the impact of any shift.

\section{ARMA predictors}

The mean shift induces negative autocorrelation in the estimated residual of $\mathrm{M}_{2}$ (see the distribution of the Durbin and Watson, 1950, $D W$ statistic in the Monte Carlo, table 3). To see why this occurs, recall that for a large enough value of $\delta_{\mu}$ the optimal value of the $\operatorname{AR}(1)$ parameter is unity when $\rho=0$, so that differencing (1) yields:

$$
\Delta y_{t}=\delta_{\mu} D_{t}+\Delta \epsilon_{t}
$$

where $D_{t}$ is an impulse dummy which is unity when $t=\tau+1$, and zero otherwise. If the break is not modelled, then we have the IMA $(1,1)$ representation of the process:

$$
\Delta y_{t}=\kappa+\xi_{t}+\theta \xi_{t-1}
$$

As $\delta_{\mu} \rightarrow 0, \theta \rightarrow-1$, so the limit is an over-differenced process, with a strictly non-invertible MA component. For $\delta_{\mu}>0$, the role of differencing is to convert the step-change in the mean of the whitenoise process $y_{t}$ into a blip in the estimated residual of the IMA representation. Figure 7 shows time series plots for a single realization of $y_{t}$ from the Monte Carlo and the estimated residual $\left(\widehat{\xi}_{t}\right)$ from $\mathbf{M}_{7}$.

For the size of break in the Monte Carlo, the AR root in $\mathrm{M}_{8}$ close to unity. In $\mathrm{M}_{6}$ and $\mathrm{M}_{7}$ (IMA $(1,1$ ) models with and without constant terms), the MA coefficient is estimated at -0.42 and -0.40 , respectively, and in $\mathrm{M}_{8}$ (ARMA $(1,1)$ ) at -0.39 . A neglected negative MA and near-unit root are conditions 

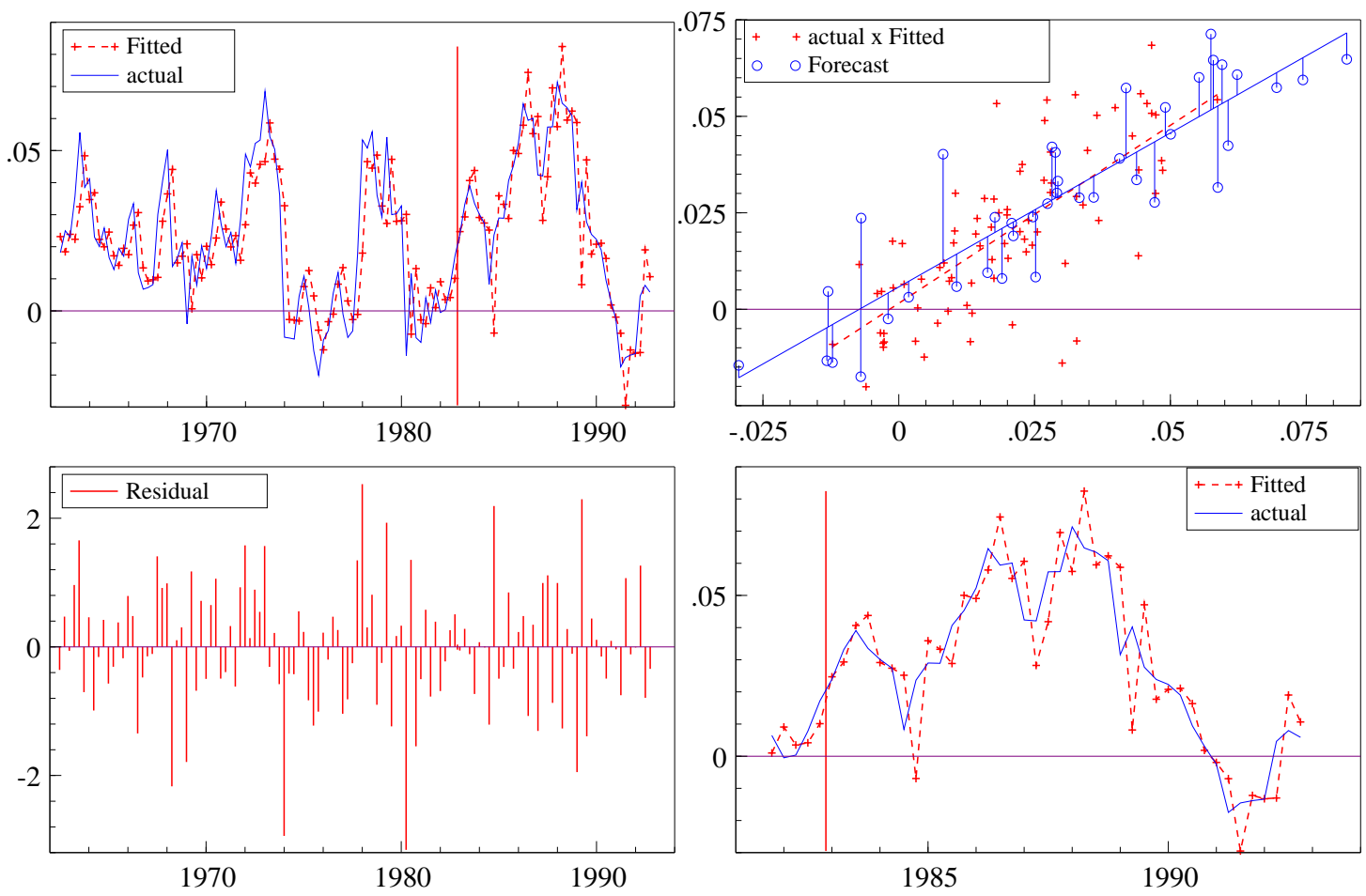

Figure 5 Graphical statistics for the intercept-corrected AR(1) model.
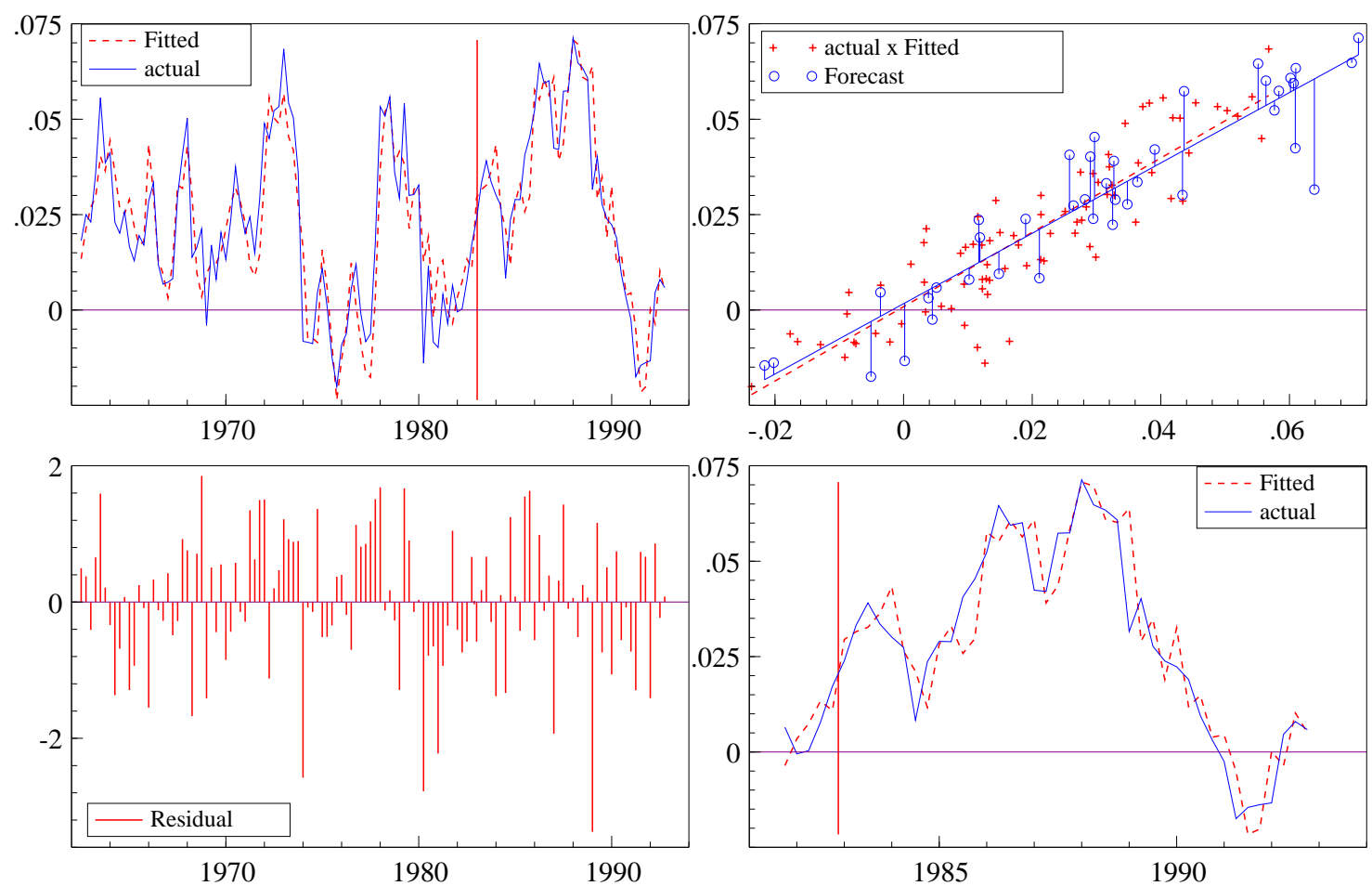

Figure 6 Graphical statistics for the intercept-corrected DHSY model.

under which the results in Clements and Hendry (1996c) suggest that multi-step estimation of purely autoregressive models, such as $\mathbf{M}_{2}$, should yield gains. We elaborate on this line of reasoning in $\S 10$. 

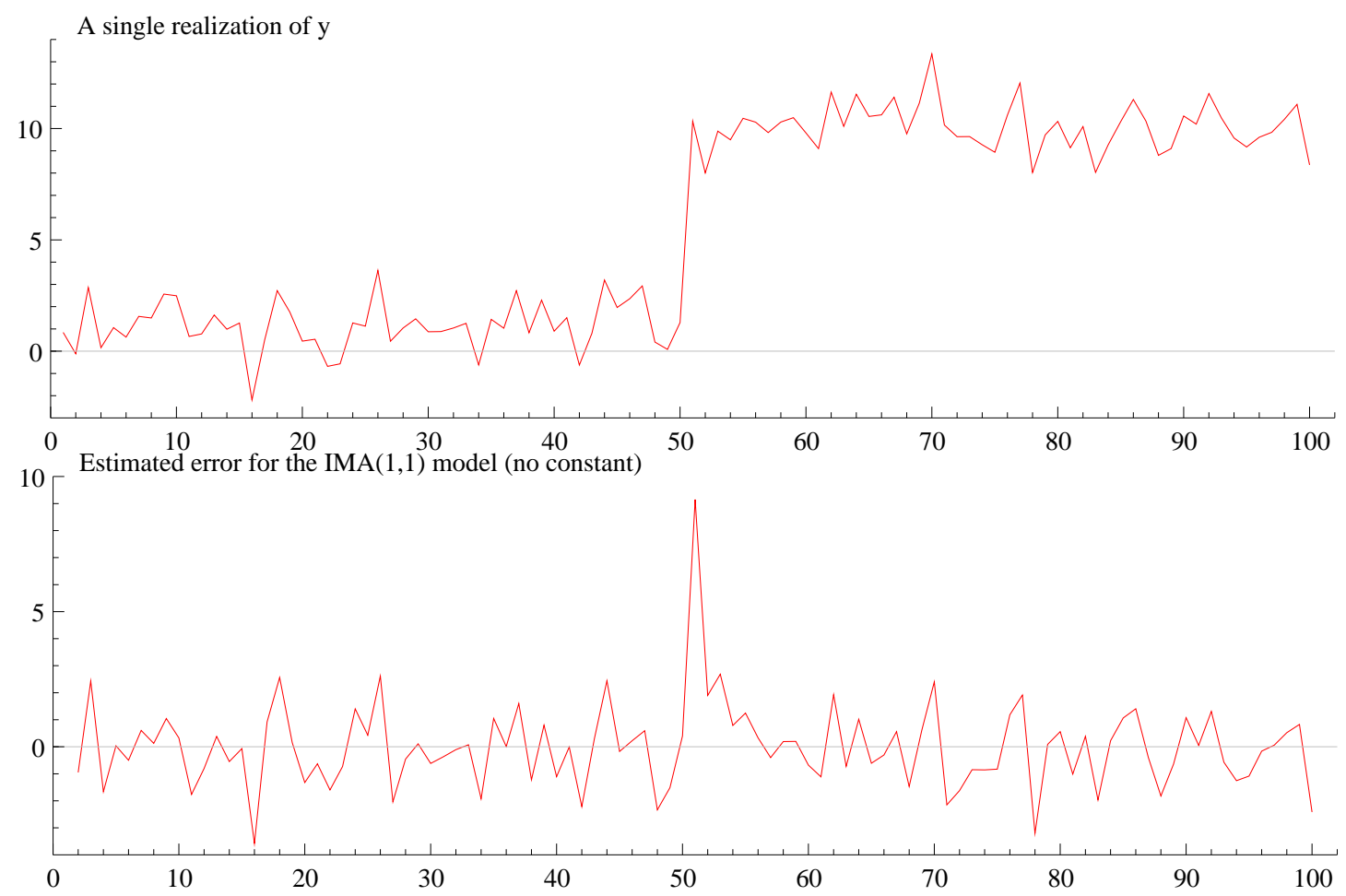

Figure 7 Time series of a single realization of $y_{t}$ with the estimated errors from $\mathrm{M}_{7}$.

The optimal forecast function for the IMA $(1,1)$ is:

$$
y_{T+h \mid T}=y_{T}+\widehat{\theta} \widehat{\xi}_{T}
$$

where $\widehat{\theta}$ and $\widehat{\xi}_{T}$ denote estimates. The bias of this predictor is zero:

$$
\mathrm{E}\left[y_{T+h}-y_{T+h \mid T}\right]=\mu_{2}-\mu_{2}-\widehat{\theta} \mathrm{E}\left[\widehat{\xi}_{T}\right]=0 .
$$

since $\mathrm{E}\left[\widehat{\xi}_{t}\right]=0$ except at $t=\tau+1$, where it picks up the effect of the mean shift. The forecast error variance is approximately (ignoring the fact that $\widehat{\theta}$ is a random variable):

$$
\mathrm{E}\left[\left(y_{T+h}-y_{T+h \mid T}\right)^{2}\right]=\mathrm{E}\left[\left(\epsilon_{T+h}-\epsilon_{T}-\widehat{\theta}_{T}\right)^{2}\right] \simeq 2 \sigma_{\epsilon}^{2}+\widehat{\theta}^{2} \mathrm{E}\left[\widehat{\xi}_{T}^{2}\right]+2 \widehat{\theta} \mathrm{E}\left[\widehat{\xi}_{T} \epsilon_{T}\right]
$$

We have:

$$
\widehat{\xi}_{T}=y_{T}-y_{T-1}-\widehat{\theta} \widehat{\xi}_{T-1}=\Delta \epsilon_{T}-\widehat{\theta} \widehat{\xi}_{T-1}=\Delta \epsilon_{T}-\widehat{\theta}\left(\Delta \epsilon_{T-1}-\widehat{\theta} \widehat{\xi}_{T-2}\right)
$$

so that $\mathrm{E}\left[\widehat{\xi}_{T} \epsilon_{T}\right] \simeq \sigma_{\epsilon}^{2}$. Also, from (13):

$$
\widehat{\xi}_{T}=\epsilon_{T}-(1+\widehat{\theta}) \epsilon_{T-1}+\widehat{\theta}(1+\widehat{\theta}) \epsilon_{T-2}-\cdots
$$

so:

$$
\mathrm{E}\left[\widehat{\xi}_{T}^{2}\right]=\sigma_{\epsilon}^{2}\left[1+(1+\widehat{\theta})^{2} \sum_{i=0}^{\infty} \widehat{\theta}^{2 i}\right] \simeq \sigma_{\epsilon}^{2} \frac{2(\widehat{\theta}+1)}{1-\widehat{\theta}^{2}}
$$


(effectively assuming that the structural break occurred sufficiently far back to be ignored). Then:

$$
\mathrm{E}\left[\left(y_{T+h}-y_{T \mid T+h}\right)^{2}\right]=\sigma_{\epsilon}^{2}\left(2+2 \theta+\frac{2 \widehat{\theta}^{2}(\widehat{\theta}+1)}{1-\widehat{\theta}^{2}}\right)=\frac{2 \sigma_{\epsilon}^{2}}{1-\widehat{\theta}}
$$

yielding a forecast error variance less than that for the differenced-model (for $\theta<0$ ). This result is unsurprising since the IMA model fits the data better than the $\operatorname{ARIMA}(0,1,0)$ (due to its negative serial correlation). In terms of bias, the IMA $\left(\mathrm{M}_{7}\right)$ forecasts are similar to the differenced-model $\left(\mathrm{M}_{5}\right)$ forecasts since the expected value of the MA term in the forecast function is zero.

As before, the empirical results conform to these analytic predictions. First, as there is no first-order residual autocorrelation in (6), but there is fourth, we estimated an ARMA $(1,4)$ where only the lag-four error is included. This yielded the estimates in (14), and the graphical outcomes in Figure 8:

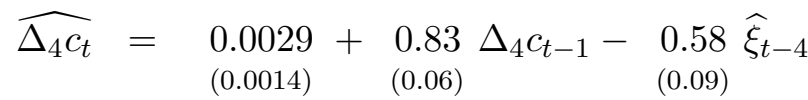

$$
\begin{aligned}
& \mathrm{R}^{2}=0.66 \widehat{\sigma}_{\xi}=1.22 \% \mathrm{~F}_{\mathrm{ar}}(4,73)=0.53 \quad \mathrm{~F}_{\text {arch }}(4,69)=0.44 \\
& \chi_{\text {nd }}^{2}(2)=0.93 \mathrm{~F}_{\text {het }}(4,72)=3.20^{* *} \mathrm{SC}=-8.69
\end{aligned}
$$
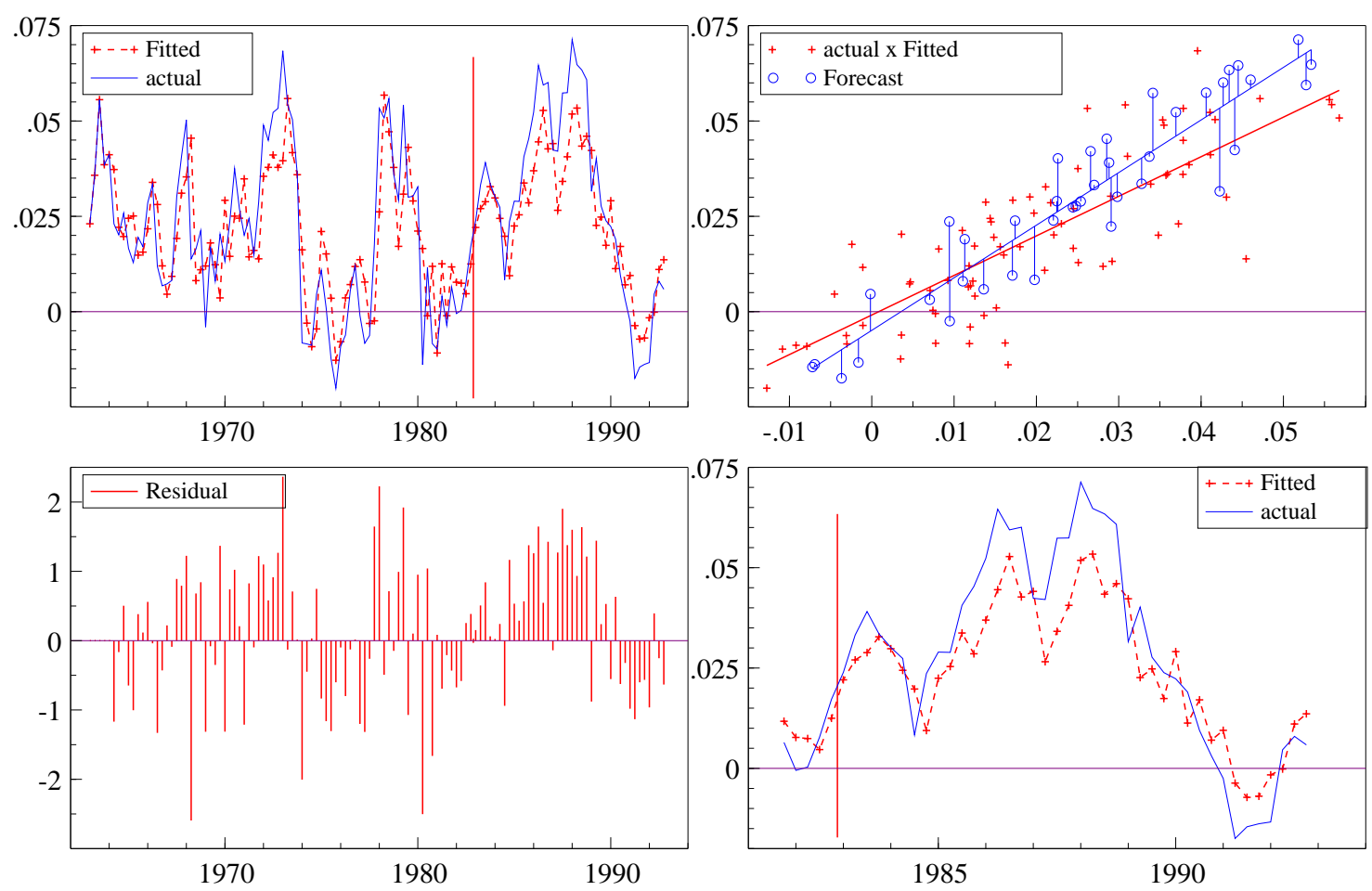

Figure 8 Graphical statistics for the ARMA $(1,4)$ model.

The forecasts remain poor, although the residual autocorrelation has vanished. This is in contrast to imposing a zero intercept and unit root on $\Delta_{4} c_{t-1}$ (when $\widehat{\sigma}=1.26 \%$ and $\mathrm{SC}=-8.71$ ): see Figure 9 .

\section{AR(1) model - multi-step estimation}

Multi-step estimation may result improve forecast accuracy whenever the parameter defined by projection of $y_{T+h}$ on to $y_{T}$ is not $h$ times the parameter defined by the 1-step projection, that is, when 

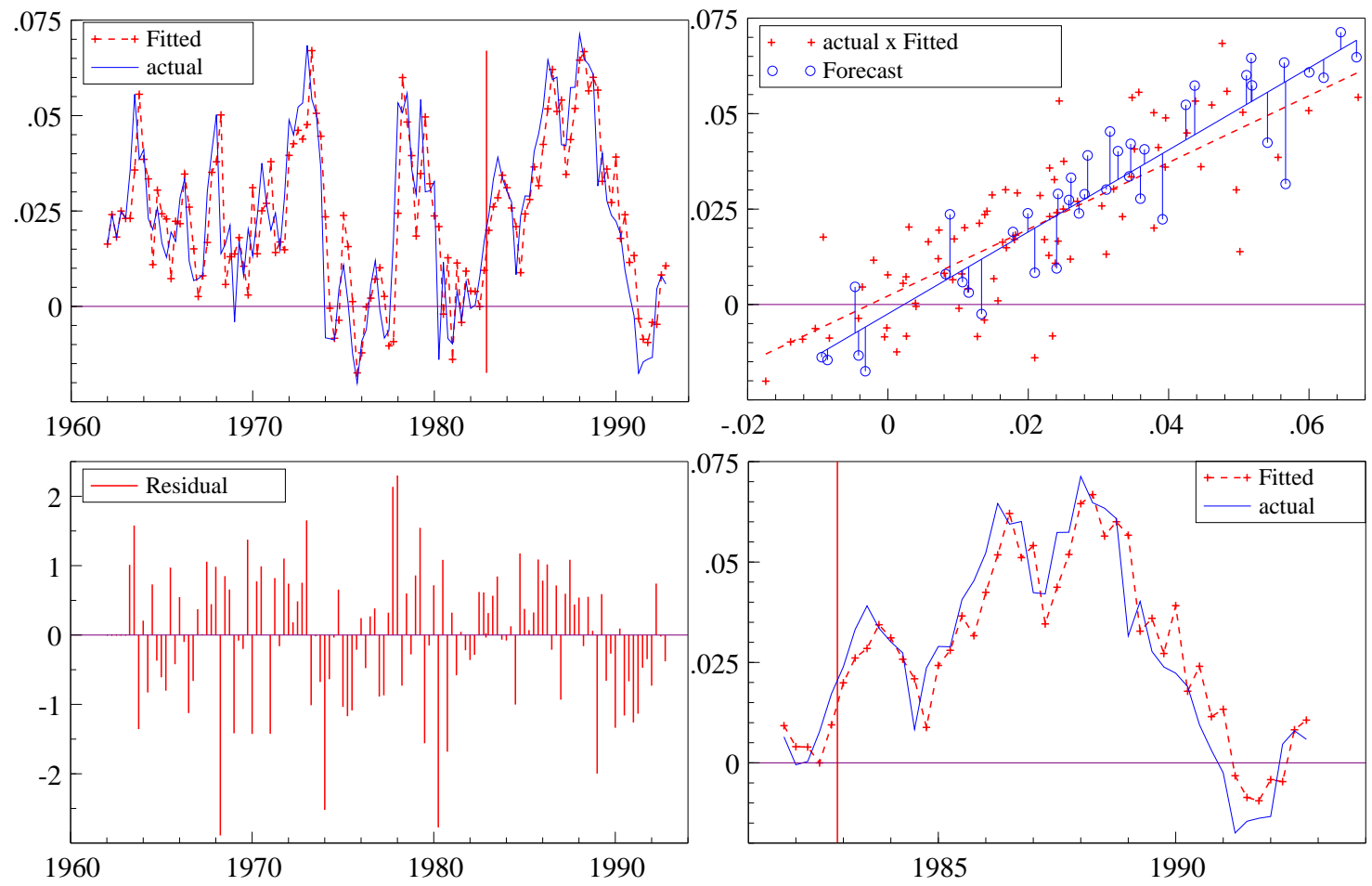

Figure 9 Graphical statistics for the IMA $(1,4)$ model.

$\mathrm{E}\left[y_{T+1} \mid y_{T}\right]=\psi y_{T}$ say, but $\mathrm{E}\left[y_{T+h} \mid y_{T}\right]=\psi_{h} y_{T} \neq \psi^{h} y_{T}$ : see Clements and Hendry (1996c). Then, however accurately $\widehat{\psi}$ estimates $\psi,(\widehat{\psi})^{h}$ need not be close to $\psi_{h}$. In the case we consider, this discrepancy arises because of the mean shift inducing a negative MA term, which is omitted from $\mathrm{M}_{2}$. Thus, when $\kappa=0$ in (12) and no intercept is estimated:

$$
\mathrm{E}\left[y_{T+h} \mid y_{T}\right]=\rho^{h} y_{T}+\rho^{h-1} \theta \mathrm{E}\left[\xi_{T} \mid y_{T}\right]=\rho^{h-1}(\rho+\theta \lambda) y_{T}=\rho^{h-1} \phi y_{T}
$$

where $\mathrm{E}\left[\xi_{T} \mid y_{T}\right]=\lambda y_{T}$. Consequently, $\psi=(\rho+\theta \lambda)$, but $\psi_{h}=\rho^{h-1} \psi \neq \psi^{h}$ unless $\theta=0$. We can handle $\kappa \neq 0$ with a change of notation, and for models of arbitrary lag order, this analysis is applicable to their first-order companion forms. For example, for $\kappa \neq 0$ write $\mathbf{y}_{t}=\left(y_{t}: 1\right)^{\prime}$ with $\mathbf{v}_{t}=\boldsymbol{\xi}_{t}+\boldsymbol{\theta} \boldsymbol{\xi}_{t-1}$ where $\boldsymbol{\xi}_{t}=\left(\xi_{t}: 0\right)^{\prime}$, so that:

$$
\boldsymbol{\rho}=\left[\begin{array}{cc}
\rho & \kappa \\
0 & 1
\end{array}\right] \text { and } \boldsymbol{\theta}=\left[\begin{array}{cc}
\theta & 0 \\
0 & 1
\end{array}\right]
$$

then:

$$
\mathbf{y}_{t}=\boldsymbol{\rho} \mathbf{y}_{t-1}+\boldsymbol{\xi}_{t}+\boldsymbol{\theta} \boldsymbol{\xi}_{t-1} .
$$

Clements and Hendry (1996c) consider the role of multi-step estimation in such a model both when $\rho=1$ and $|\rho|<1$. For the stationary case with $\kappa=0$, they show that the optimal value of $\psi_{h}$ in the $\mathrm{AR}$ ( 1 ) model $h$-step forecast function (in the sense of minimizing $h$-step ahead MSFE) is:

$$
y_{T+h \mid T}=\psi_{h} y_{T}
$$

is given by:

$$
\psi_{h} \equiv \rho_{\{h\}}=\rho^{(h-1)} \times \rho_{\{1\}}
$$

where $\rho_{\{1\}}$ is optimal for 1-step ahead forecast errors, and is given by: 


$$
\rho_{\{1\}}=\rho+\frac{\theta\left(1-\rho^{2}\right)}{1+\theta^{2}+2 \rho \theta},
$$

from which it is apparent that $\psi_{h}=\psi^{h} \equiv\left(\rho_{\{1\}}\right)^{h}$ requires that $\theta=0$. When $\rho=1$ :

$$
\rho_{\{h\}}=\left(\rho_{\{1\}}\right)^{1 / h}
$$

so that $\rho_{\{h\}} \rightarrow 1$ as $h$ increases (when $\theta>-1$ ). Notice that $\psi^{h} \equiv\left(\rho_{\{1\}}\right)^{h} \rightarrow 0$ in $h$ when $\left|\rho_{\{1\}}\right|<1$.

In the Monte Carlo, multi-step estimation inflates the forecast error variances over 1-step estimation, but generates smaller biases and an overall gain in terms of MSFE (compare the column headed $\mathrm{M}_{3}$ with $\mathrm{M}_{2}$ in table 2). For $\rho=1$, the limiting distribution of $\widehat{\psi_{h}}$ is closely related to that of $\widehat{\psi}$ (see Clements and Hendry, 1996c), and predicts that biases remain and do not change much with $h$. However, different estimators are required for each linear transform of the dependent variable (e.g., $\Delta_{4} c_{t}$ versus $\Delta_{1} \Delta_{4} c_{t}$ even though $\left.\Delta_{1} \Delta_{4} c_{t} \equiv \Delta_{1} c_{t}-\Delta_{1} c_{t-4}\right)$.

Here we compare 4-step estimation for 4-period ahead forecasts with fourth powering of the 1step form. The AR (1) given by (6) provides 4 -step ahead forecasts of $\Delta_{4} c_{t}$ by powering up. Direct estimation of the 4-step representation yields:

$$
\begin{aligned}
\widehat{\Delta_{4} c_{t}} & =\underset{(0.0033)}{0.017}+\underset{(0.11)}{0.14 \Delta_{4} c_{t-4}} \\
\mathrm{R}^{2} & =0.02 \widehat{\sigma}_{\varsigma}=2.08 \% \mathrm{~J}=1.82 \mathrm{SC}=-7.66
\end{aligned}
$$

This is for the annual growth in $c_{t}$ one year ahead, and reveals almost no forecastability from the previous year's annual growth. Few tests are valid due to the residual autocorrelation, which also biases the conventional standard errors. The fourth power of 0.74 (the slope coefficient in (6)) is 0.30 and imposing that yields $\widetilde{\sigma}_{\varsigma}=2.09 \%$ : Figure 10 shows the comparison of the residuals and forecasts. There is little to choose between the two estimators, but the powered-up forecasts are somewhat better here with MSFEs of $2.4 \%$ versus $2.75 \%$. In absolute terms, neither would be of use for economic policy, with forecast errors of $4 \%$ occurring regularly.

The situation is more sanguine for quarterly growth one-year ahead. Using the identity noted above, $\Delta_{1} c_{t} \equiv \Delta_{1} \Delta_{4} c_{t}+\Delta_{1} c_{t-4}$ and as $\Delta_{1} \Delta_{4} c_{t}$ can be explained in part as $-\frac{1}{2} \Delta_{1} \Delta_{4} c_{t-4}$ we have (unrestricted estimates are $0.98(0.03)$ and $-0.43(0.10))$ :

$$
\begin{aligned}
\widehat{\Delta_{1} c_{T+4}} & =\Delta_{1} c_{T}-\frac{1}{2} \Delta_{1} \Delta_{4} c_{T} \\
\mathrm{R}^{2} & =0.95 \widehat{\sigma}_{\zeta}=1.36 \% \quad \mathrm{SC}=-8.55
\end{aligned}
$$

Figure 11 shows the forecasts with conventionally-calculated $95 \%$ confidence intervals.

This overestimates the uncertainty from the policy-makers perspective, since tax changes are known to them, so to establish a 'minimum' innovation variance, we added indicator variables for the main changes in consumers' taxes (purchase tax in 1968(1)/(2); VAT in 1973(1)/(2) and 1979(2)/(3)), and interventions in 1974(1), 1977(4)/1978(1) and 1980(1) for the remaining outliers. Then $\widetilde{\sigma}_{\zeta}=0.93 \%$ and Figure 12 shows the graphical statistics in terms of $\Delta_{1} \Delta_{4} c_{t}$, with $95 \%$ confidence bands rather than bars.

There is only one significant forecast error, and the MSFE is just under $0.9 \%$. However, it is hard to decide what the 1-step comparative method would be, since models of $\Delta_{1} \Delta_{4} c_{t}$ primarily depend on 

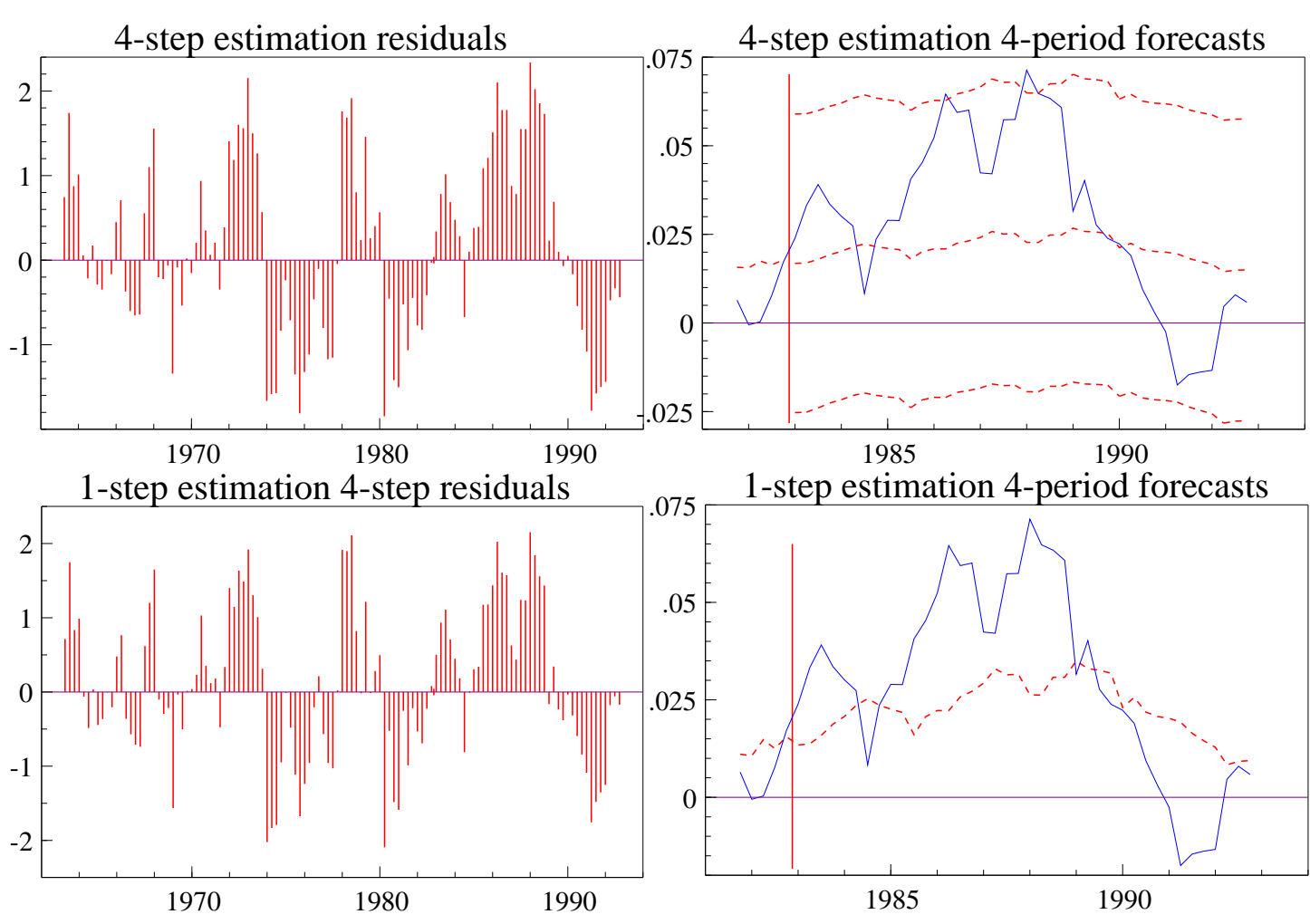

Figure 10 Graphical statistics for 4-step ahead forecasts of annual growth.

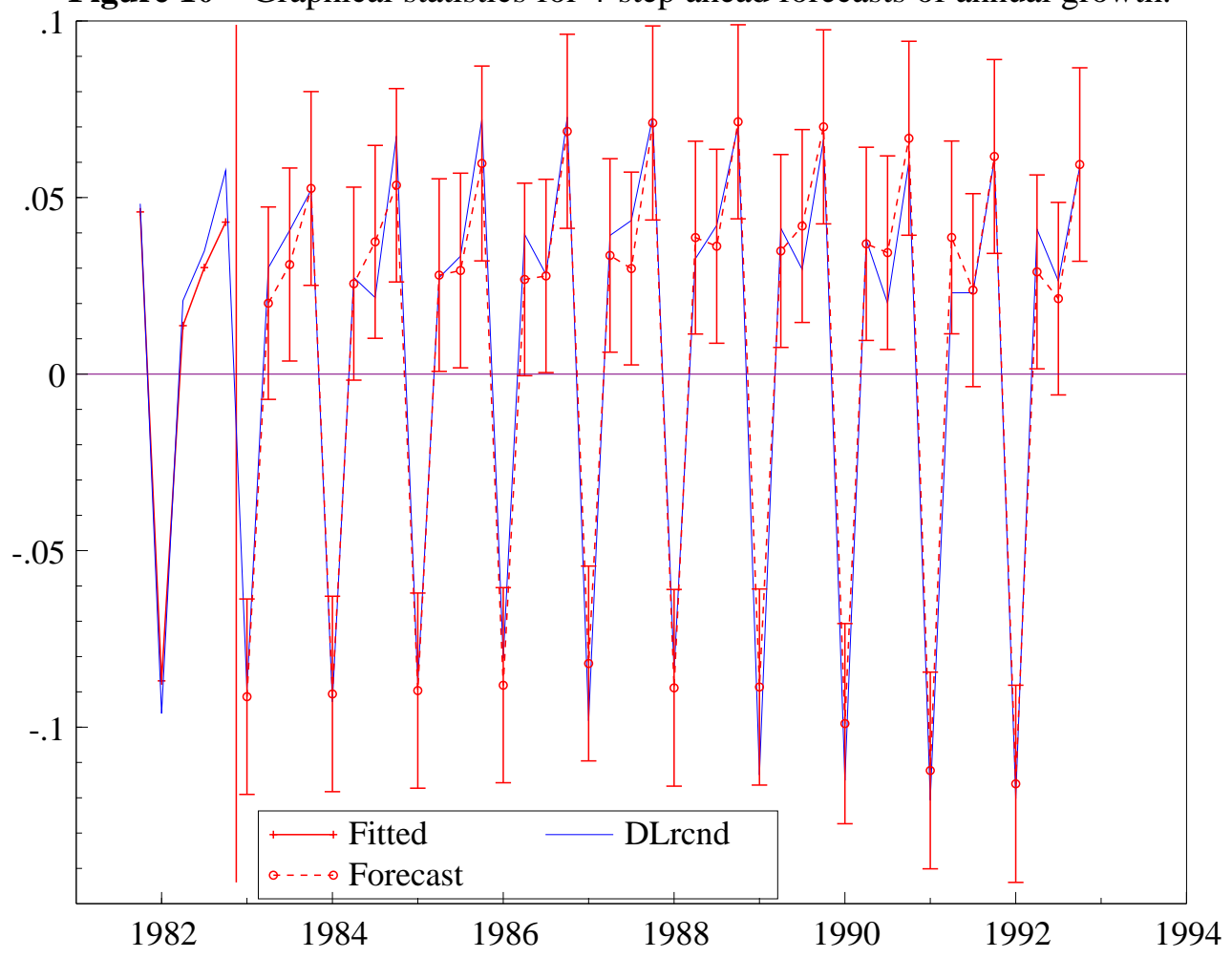

Figure 11 4-step ahead forecasts of quarterly growth.

$\Delta_{1} \Delta_{4} c_{t-4}$. Finally, Figure 13 shows the forecasts from 4-7 steps ahead for $\Delta_{1} c_{t}$ based on the 'models':

$$
\widehat{\Delta_{1} c_{t}}=\widehat{\alpha}_{4}+\Delta_{1} c_{t-4}-0.5 \Delta_{1} \Delta_{4} c_{t-4}
$$



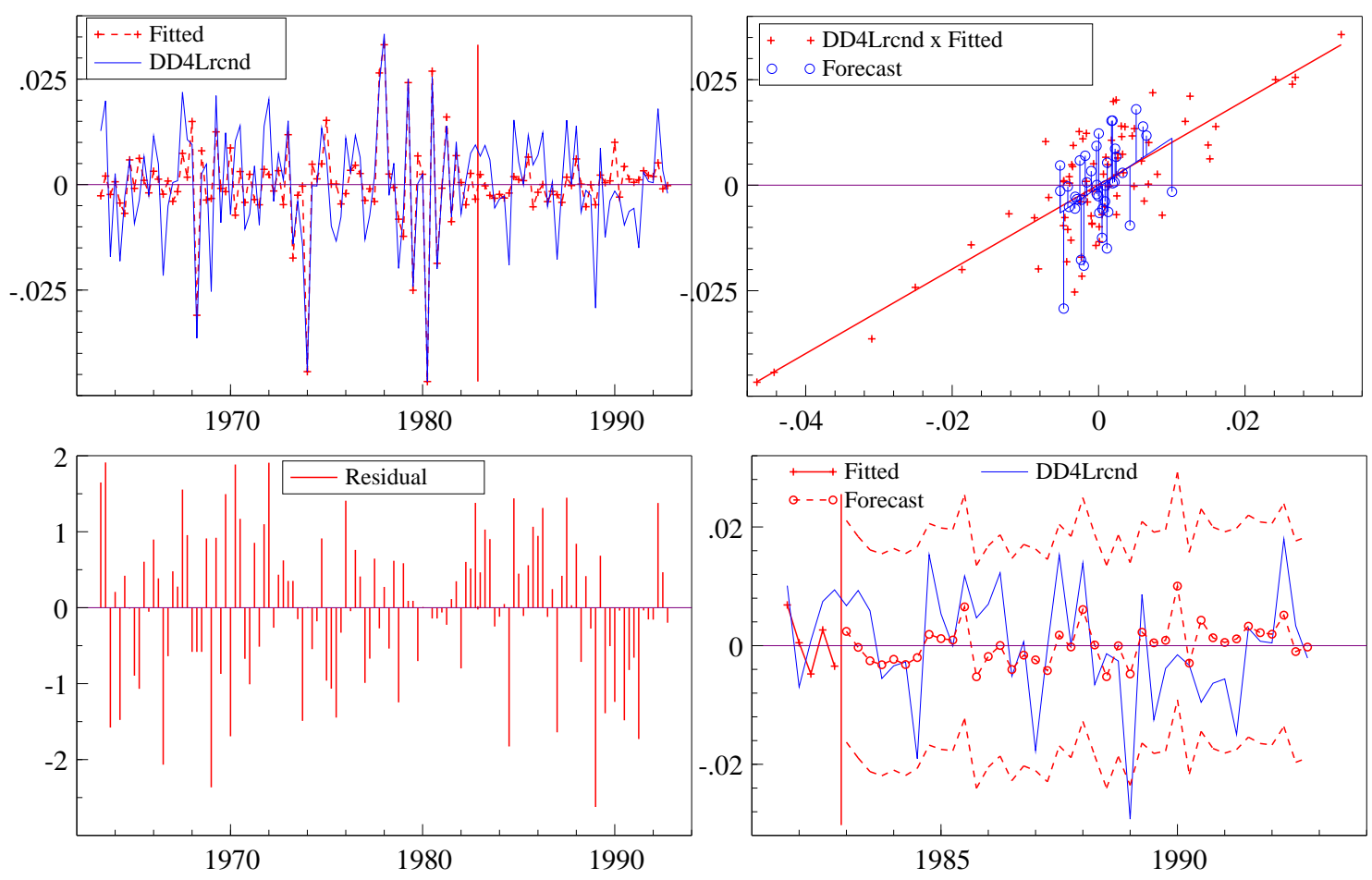

Figure 12 Graphical statistics for 4-step ahead forecasts of quarterly changes in annual growth.

$$
\begin{aligned}
& \widehat{\Delta_{1} c_{t}}=\widehat{\alpha}_{5}+\Delta_{1} c_{t-8}-0.25\left(\Delta_{4} c_{t-5}\right) \\
& \widehat{\Delta_{1} c_{t}}=\widehat{\alpha}_{6}+\Delta_{1} c_{t-8}-0.33\left(\Delta_{4} c_{t-6}\right) \\
& \widehat{\Delta_{1} c_{t}}=\widehat{\alpha}_{7}+\Delta_{1} c_{t-8}-0.33\left(\Delta_{4} c_{t-7}\right)
\end{aligned}
$$

where only the $\alpha_{i}$ are estimated. There is not a great deal of deterioration as the horizon increases, the sample and forecast statistics being:

$\begin{array}{ccccc}i & 100 \widehat{\alpha_{i}} & 100 \mathrm{SE} & 100 \widehat{\sigma_{i}} & \chi_{i}^{2}(40) \\ 4 & -0.01 & 0.15 & 1.36 & 17.9 \\ 5 & 0.50 & 0.17 & 1.52 & 20.2 \\ 6 & 0.64 & 0.17 & 1.47 & 23.9 \\ 7 & 0.64 & 0.17 & 1.47 & 22.6\end{array}$

where:

$$
\chi_{i}^{2}(40)=\frac{1}{\widehat{\sigma_{i}^{2}}} \sum_{t=T+1}^{T+40}\left(\Delta_{1} c_{t}-\widehat{\Delta_{1} c_{t}}\right)^{2}=\left(40 / \widehat{\sigma_{i}^{2}}\right) \times \text { MSFE }
$$

Thus, in every case, the average forecast errors are about half the in-sample.

\section{Disequilibrium adjustment}

When the process is (1) but with $\rho \neq 0$, that is:

$$
y_{t}=\rho y_{t-1}+\mu^{*}+\epsilon_{t}
$$



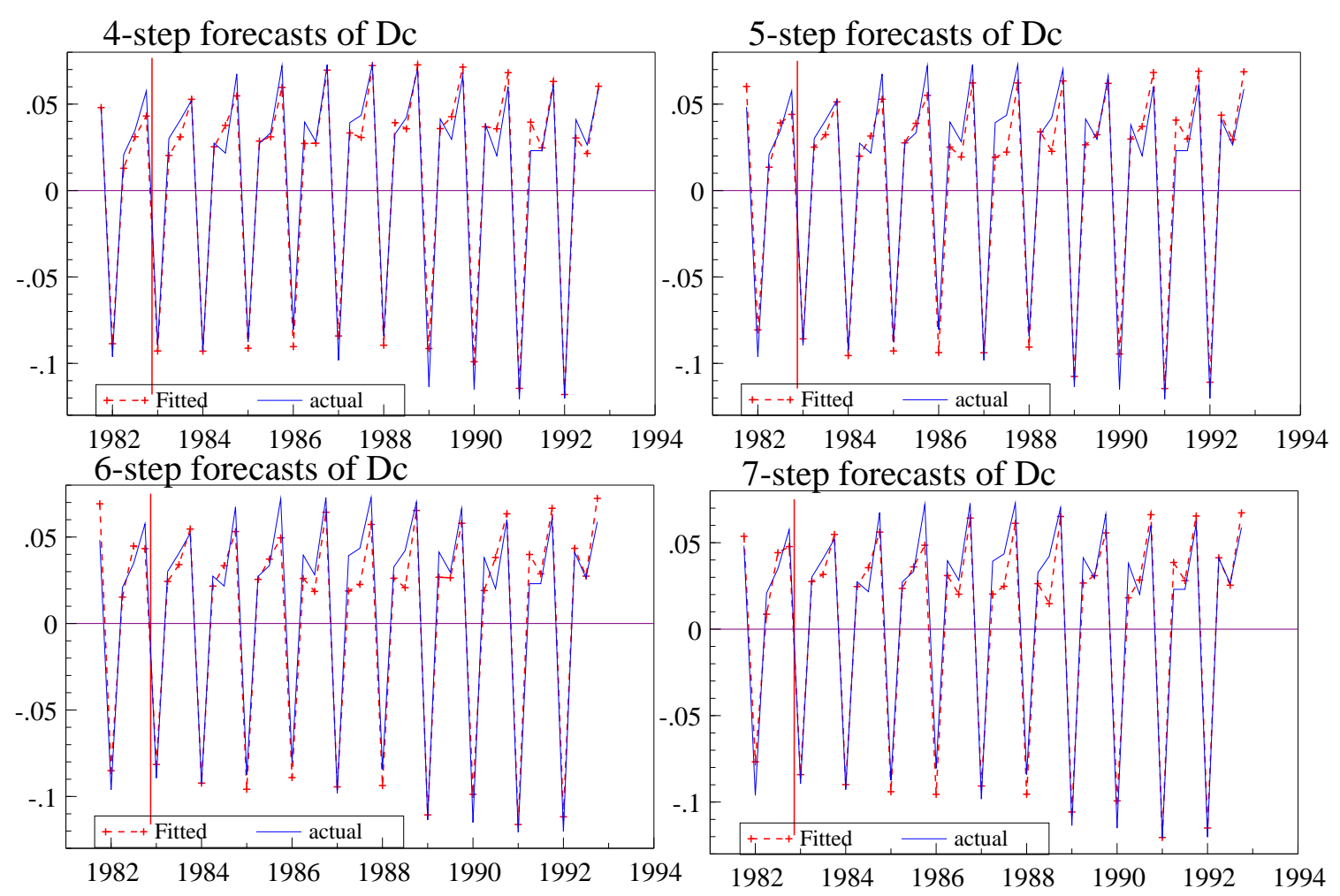

Figure 13 4-step to 7-step ahead forecasts of quarterly growth.

then there will be a period of adjustment when the mean of $y$ alters from $\frac{\mu_{1}}{1-\rho}$ to $\frac{\mu_{2}}{1-\rho}$, since:

$$
y_{t}=\mu_{2} \sum_{s=0}^{t-\tau-1} \rho^{s}+\mu_{1} \sum_{s=t-\tau}^{t-1} \rho^{s}+\sum_{s=0}^{t-1} \rho^{s} \epsilon_{t-s}+\rho^{t} y_{0}
$$

for $t>\tau+1$. When $y_{0} \sim \mathrm{D}\left(\frac{\mu_{1}}{1-\rho}, \frac{\sigma_{\epsilon}^{2}}{1-\rho^{2}}\right)$, then for $t \leq \tau, \mathrm{E}\left[y_{t}\right]=\frac{\mu_{1}}{1-\rho}$. For $t>\tau, \mathrm{E}\left[y_{t}\right] \rightarrow \frac{\mu_{2}}{1-\rho}$ as $t \rightarrow \infty$. The speed of adjustment depends on how close $\rho$ is to zero: at $\rho=0$ the adjustment is instantaneous. If, as in the Monte Carlo, $\tau=\frac{T}{2}$ and $T$ is relatively large, then forecasting from an origin of $T$ will yield similar results to when $\rho=0$. Since $\mathrm{E}\left[y_{T}\right] \simeq \mu_{2}$, the latest observation (nearly) fully embodies the new equilibrium mean so the differenced-model $\mathrm{M}_{5}$ forecasts will be approximately unbiased.

Reconsider (1) pre-break, written as:

$$
\Delta y_{t}=(\rho-1)\left(y_{t-1}-\frac{\mu_{1}}{1-\rho}\right)+v_{t} .
$$

The disequilibrium:

$$
y_{t-1}-\frac{\mu_{1}}{1-\rho}=y_{t-1}-\mathrm{E}\left[y_{t-1}\right]
$$

helps correct the current growth $\Delta y_{t}$, and ensures a stationary outcome. However, when the long-run mean changes to $\mu_{2} /(1-\rho)$, the DGP becomes:

$$
\Delta y_{t}=(\rho-1)\left(y_{t-1}-\frac{\mu_{2}}{1-\rho}\right)+v_{t} .
$$

whereas the model in use remains:

$$
\Delta y_{t}=(\rho-1)\left(y_{t-1}-\frac{\mu_{1}}{1-\rho}\right)+v_{t}
$$




$$
=(\rho-1)\left(y_{t-1}-\frac{\mu_{2}}{1-\rho}\right)+v_{t}-\delta_{\mu}
$$

When $\delta_{\mu}>0$, as in Figure 7, the EqCM computed in (17) will be persistently positive, so will continually predict negative $\Delta y_{t+j}$. Thus, as noted in $\S 4$, this is the opposite of error-correction, and corrects only within equilibria and not between. We suspect that this underlies the failure of DHSY, namely a shift in the desired long-run propensity to spend following financial deregulation that lifted a rationing constraint on borrowing for many consumers, enabling them to finance a higher ratio of expenditure to income. Rationing ceased to bite around 1989, and the rise in interest rates, reduction of tax deductibility of interest payments, and the fall in house prices (the main collateral for the debt) together forced a return to previous levels of expenditure to income. Such mean shifts are consistent with the success of the intercept-correction strategy above.

\section{Conclusions}

The main aim of the paper was to exposit a number of recent results on forecasting after a shift in the mean of a stochastic process. When economic systems are subject to structural breaks, conventional models need not forecast satisfactorily. The empirical example of consumers' expenditure reveals that some shift in the econometric relation occurred, and that the various strategies considered helped circumvent the implicit shift. Analysis suggests that differencing can mitigate the effects of changes in equilibrium means, and this was shown above empirically, and in a Monte Carlo. Alternatively, intercept corrections can offset the mean shift, albeit at the cost of an increased variance. However, models that do neither performed badly analytically and empirically. There was little benefit to multi-step estimation over repeated backward solution of 1-step estimates.

Thus, in-sample fit may be a poor guide to ex-ante forecast performance when the assumption of constancy fails, so alternative strategies may be called for. A theory of forecasting allowing for structural breaks when the model is not the mechanism is feasible, and on the limited evidence of the empirical example considered here, provides a useful basis for interpreting and circumventing systematic predictive failure in economics.

\section{References}

Andrews, D. W. K. (1993). Tests for parameter instability and structural change with unknown change point. Econometrica, 61, 821-856.

Box, G. E. P., and Jenkins, G. M. (1976). Time Series Analysis, Forecasting and Control. San Francisco: Holden-Day.

Carruth, A., and Henley, A. (1990). Can existing consumption functions forecast consumer spending in the late 1980s?. Oxford Bulletin of Economics and Statistics, 52, 211-222.

Chow, G. C. (1964). A comparison of alternative estimators for simultaneous equations. Econometrica, 32, 532-553.

Chu, C.-S., Stinchcombe, M., and White, H. (1996). Monitoring structural change. Econometrica, 64, 1045-1065.

Clements, M. P., and Hendry, D. F. (1993). On the limitations of comparing mean squared forecast errors. Journal of Forecasting, 12, 617-637. With discussion. 
Clements, M. P., and Hendry, D. F. (1995). Macro-economic forecasting and modelling. Economic Journal, 105, 1001-1013.

Clements, M. P., and Hendry, D. F. (1996a). Forecasting in macro-economics. In Cox, D. R., Hinkley, D. V., and Barndorff-Nielsen, O. E. (eds.), Time Series Models: In econometrics, finance and other fields, pp. 101-141. London: Chapman and Hall.

Clements, M. P., and Hendry, D. F. (1996b). Intercept corrections and structural change. Journal of Applied Econometrics, 11, 475-494.

Clements, M. P., and Hendry, D. F. (1996c). Multi-step estimation for forecasting. Oxford Bulletin of Economics and Statistics, 58, 657-684.

Davidson, J. E. H., Hendry, D. F., Srba, F., and Yeo, J. S. (1978). Econometric modelling of the aggregate time-series relationship between consumers' expenditure and income in the United Kingdom. Economic Journal, 88, 661-692. Reprinted in Hendry, D. F. (1993), Econometrics: Alchemy or Science? Oxford: Blackwell Publishers.

Doornik, J. A., and Hansen, H. (1994). A practical test for univariate and multivariate normality. Discussion paper, Nuffield College.

Durbin, J., and Watson, G. S. (1950). Testing for serial correlation in least squares regression I. Biometrika, 37, 409-428.

Engle, R. F. (1982). Autoregressive conditional heteroscedasticity, with estimates of the variance of United Kingdom inflations. Econometrica, 50, 987-1007.

Fildes, R., and Makridakis, S. (1995). The impact of empirical accuracy studies on time series analysis and forecasting. International Statistical Review, 63, 289-308.

Godfrey, L. G. (1978). Testing for higher order serial correlation in regression equations when the regressors include lagged dependent variables. Econometrica, 46, 1303-1313.

Hansen, B. E. (1992). Testing for parameter instability in linear models. Journal of Policy Modeling, 14, 517-533.

Hendry, D. F. (1974). Stochastic specification in an aggregate demand model of the United Kingdom. Econometrica, 42, 559-578. Reprinted in Hendry, D. F. (1993), Econometrics: Alchemy or Science? Oxford: Blackwell Publishers.

Hendry, D. F. (1994). HUS revisited. Oxford Review of Economic Policy, 10, 86-106.

Hendry, D. F., and Clements, M. P. (1994). On a theory of intercept corrections in macro-economic forecasting. In Holly, S. (ed.), Money, Inflation and Employment: Essays in Honour of James Ball, pp. 160-182. Aldershot: Edward Elgar.

Hendry, D. F., and Doornik, J. A. (1994). Modelling linear dynamic econometric systems. Scottish Journal of Political Economy, 41, 1-33.

Hendry, D. F., and Doornik, J. A. (1996a). Empirical Econometric Modelling using PcGive 9 for Windows. London: International Thomson Business Press.

Hendry, D. F., and Doornik, J. A. (1996b). Empirical Econometric Modelling using PcGive for Windows. London: International Thomson Business Press.

Hendry, D. F., and Doornik, J. A. (1997). The implications for econometric modelling of forecast failure. Scottish Journal of Political Economy. forthcoming, Centenary Issue.

Johansen, S. (1988). Statistical analysis of cointegration vectors. Journal of Economic Dynamics and Control, 12, 231-254.

Klein, L. R. (1971). An Essay on the Theory of Economic Prediction. Chicago: Markham Publishing 
Company.

Muellbauer, J. N. J. (1994). The assessment: Consumer expenditure. Oxford Review of Economic Policy, 10, 1-41.

Muellbauer, J. N. J., and Murphy, A. (1989). Why has UK Personal Saving Collapsed? London: Credit Suisse First Boston.

Pole, A., West, M., and Harrison, J. (1994). Applied Bayesian Forecasting and Time Series Analysis. London: Chapman and Hall.

Ramsey, J. B. (1969). Tests for specification errors in classical linear least squares regression analysis. Journal of the Royal Statistical Society B, 31, 350-371.

Turner, D. S. (1990). The role of judgement in macroeconomic forecasting. Journal of Forecasting, 9 , 315-345.

Wallis, K. F. (1989). Macroeconomic forecasting: A survey. Economic Journal, 99, 28-61.

Wallis, K. F., Andrews, M. J., Fisher, P. G., Longbottom, J., and Whitley, J. D. (1986). Models of the UK Economy: A Third Review by the ESRC Macroeconomic Modelling Bureau. Oxford: Oxford University Press.

Wallis, K. F., Fisher, P. G., Longbottom, J., Turner, D. S., and Whitley, J. D. (1987). Models of the UK Economy: A Fourth Review by the ESRC Macroeconomic Modelling Bureau. Oxford: Oxford University Press.

Wallis, K. F., and Whitley, J. D. (1991). Sources of error in forecasts and expectations: U.K. economic models 1984-8. Journal of Forecasting, 10, 231-253.

West, M., and Harrison, P. J. (1989). Bayesian Forecasting and Dynamic Models. New York: Springer Verlag.

White, H. (1980). A heteroskedastic-consistent covariance matrix estimator and a direct test for heteroskedasticity. Econometrica, 48, 817-838.

\section{Monte Carlo tables}


Table 2 Measures of Forecast Accuracy.

\begin{tabular}{|l|c|c|c|c|c|c|c|c|c|}
\hline & $\mathrm{M}_{2}$ & $\mathrm{M}_{2}^{I C}$ & $\mathrm{M}_{3}$ & $\mathrm{M}_{3}^{I C}$ & $\mathrm{M}_{4}$ & $\mathrm{M}_{5}$ & $\mathrm{M}_{6}$ & $\mathrm{M}_{7}$ & $\mathrm{M}_{8}$ \\
\hline & \multicolumn{10}{|c|}{ MSFEs } \\
\hline 1 & 1.93 & 5.56 & 1.93 & 5.56 & 2.01 & 1.98 & 1.45 & 1.42 & 1.44 \\
2 & 1.95 & 12.02 & 1.88 & 5.38 & 2.02 & 1.95 & 1.47 & 1.39 & 1.46 \\
3 & 2.07 & 20.75 & 1.84 & 5.20 & 2.06 & 1.94 & 1.51 & 1.39 & 1.51 \\
4 & 2.31 & 31.47 & 1.86 & 5.26 & 2.20 & 1.99 & 1.64 & 1.43 & 1.62 \\
\hline & \multicolumn{10}{|c|}{ Squared biases } \\
\hline 1 & 0.04 & 0.00 & 0.04 & 0.00 & 0.01 & 0.00 & 0.03 & 0.00 & 0.02 \\
2 & 0.16 & 0.00 & 0.04 & 0.00 & 0.03 & 0.00 & 0.06 & 0.00 & 0.06 \\
3 & 0.36 & 0.00 & 0.05 & 0.00 & 0.06 & 0.00 & 0.11 & 0.00 & 0.13 \\
4 & 0.59 & 0.00 & 0.05 & 0.00 & 0.12 & 0.00 & 0.18 & 0.00 & 0.20 \\
\hline & \multicolumn{10}{|c|}{ Forecast Error Variances } \\
\hline 1 & 1.89 & 5.56 & 1.89 & 5.56 & 2.00 & 1.98 & 1.42 & 1.42 & 1.42 \\
2 & 1.79 & 12.02 & 1.83 & 5.38 & 1.99 & 1.95 & 1.40 & 1.39 & 1.39 \\
3 & 1.71 & 20.75 & 1.79 & 5.20 & 2.00 & 1.94 & 1.41 & 1.39 & 1.38 \\
4 & 1.72 & 31.47 & 1.81 & 5.26 & 2.08 & 1.99 & 1.46 & 1.43 & 1.41 \\
\hline
\end{tabular}

Table 3 Empirical distributions of DW from OLS.

\begin{tabular}{|c|c|c|c|c|c|c|c|c|c|c|}
\hline & & \multicolumn{10}{|c}{ Percentiles } \\
Mean & Std. Dev. & 1 & 5 & 10 & 25 & 50 & 75 & 90 & 95 & 99 \\
\hline 2.5980 & 0.0200 & 2.25 & 2.36 & 2.41 & 2.50 & 2.60 & 2.70 & 2.78 & 2.83 & 2.90 \\
\hline
\end{tabular}


Table 4 Empirical distributions of model parameter estimates.

\begin{tabular}{|c|c|c|c|c|c|c|c|c|c|c|c|}
\hline & & \multicolumn{1}{|c|}{ Percentiles } \\
Model & Mean & Std. Dev. & 1 & 5 & 10 & 25 & 50 & 75 & 90 & 95 & 99 \\
\hline $\mathrm{M}_{2} \beta$ & 0.9338 & 0.0001 & 0.91 & 0.92 & 0.92 & 0.93 & 0.93 & 0.94 & 0.95 & 0.95 & 0.95 \\
$\mathrm{M}_{2} \alpha$ & 0.4518 & 0.0034 & 0.33 & 0.36 & 0.38 & 0.41 & 0.45 & 0.49 & 0.53 & 0.55 & 0.61 \\
$\mathrm{M}_{2} \beta^{2}$ & 0.8721 & 0.0004 & 0.82 & 0.84 & 0.85 & 0.86 & 0.87 & 0.89 & 0.90 & 0.90 & 0.91 \\
$\mathrm{M}_{2} \beta^{3}$ & 0.8145 & 0.0007 & 0.74 & 0.77 & 0.78 & 0.80 & 0.82 & 0.83 & 0.85 & 0.86 & 0.87 \\
$\mathrm{M}_{2} \beta^{4}$ & 0.7609 & 0.0011 & 0.68 & 0.70 & 0.72 & 0.74 & 0.76 & 0.78 & 0.80 & 0.81 & 0.83 \\
\hline $\mathrm{M}_{3} \psi_{2}$ & 0.9148 & 0.0001 & 0.88 & 0.89 & 0.90 & 0.91 & 0.92 & 0.92 & 0.93 & 0.93 & 0.94 \\
$\mathrm{M}_{3}$ const. & 0.6446 & 0.0048 & 0.50 & 0.54 & 0.56 & 0.60 & 0.64 & 0.69 & 0.74 & 0.76 & 0.82 \\
$\mathrm{M}_{3} \psi_{3}$ & 0.8956 & 0.0002 & 0.86 & 0.87 & 0.88 & 0.89 & 0.90 & 0.91 & 0.91 & 0.92 & 0.93 \\
$\mathrm{M}_{3}$ const. & 0.8380 & 0.0061 & 0.67 & 0.71 & 0.74 & 0.78 & 0.83 & 0.89 & 0.94 & 0.97 & 1.03 \\
$\mathrm{M}_{3} \psi_{4}$ & 0.8766 & 0.0002 & 0.84 & 0.85 & 0.86 & 0.87 & 0.88 & 0.89 & 0.90 & 0.90 & 0.91 \\
$\mathrm{M}_{3}$ const. & 1.0307 & 0.0071 & 0.85 & 0.90 & 0.92 & 0.97 & 1.03 & 1.09 & 1.14 & 1.18 & 1.23 \\
\hline $\mathrm{M}_{4}$ const. & 0.0907 & 0.0002 & 0.06 & 0.07 & 0.07 & 0.08 & 0.09 & 0.10 & 0.11 & 0.11 & 0.12 \\
\hline $\mathrm{M}_{6} \theta$ & -0.4195 & 0.0042 & -0.54 & -0.51 & -0.50 & -0.46 & -0.43 & -0.38 & -0.33 & -0.31 & -0.24 \\
$\mathrm{M}_{6}$ const. & 0.0922 & 0.0001 & 0.07 & 0.08 & 0.08 & 0.09 & 0.09 & 0.10 & 0.10 & 0.11 & 0.11 \\
\hline $\mathrm{M}_{7} \theta$ & -0.4040 & 0.0039 & -0.53 & -0.50 & -0.48 & -0.45 & -0.41 & 0.37 & -0.32 & -0.29 & -0.23 \\
\hline $\mathrm{M}_{8} \rho$ & 0.9780 & 0.0000 & 0.97 & 0.97 & 0.97 & 0.98 & 0.98 & 0.98 & 0.98 & 0.98 & 0.99 \\
$\mathrm{M}_{8} \theta$ & -0.3884 & 0.0043 & -0.51 & -0.48 & -0.47 & -0.43 & -0.40 & -0.35 & -0.30 & -0.27 & -0.20 \\
$\mathrm{M}_{8}$ const. & 5.4986 & 0.0547 & 4.95 & 5.11 & 5.20 & 5.34 & 5.50 & 5.65 & 5.80 & 5.88 & 6.04 \\
\hline
\end{tabular}

\section{Appendix: Vector representations}

\section{1 $\mathrm{M}_{2}$}

Clements and Hendry (1996b) consider a vector of $n$ variables, denoted by $\mathbf{w}_{t}$, and represented by a first-order VAR which includes a constant and a linear deterministic trend (omitted here) :

$$
\mathbf{w}_{t}=\boldsymbol{\kappa}+\boldsymbol{\Gamma} \mathbf{w}_{t-1}+\boldsymbol{\nu}_{t}
$$

where $\boldsymbol{\nu}_{t} \sim \mathrm{IN}_{n}(\mathbf{0}, \boldsymbol{\Omega})$. The system is assumed to be integrated, and to satisfy $r<n$ cointegration relations such that (see, for example, Johansen, 1988):

$$
\boldsymbol{\Gamma}=\mathbf{I}_{n}+\boldsymbol{\alpha} \boldsymbol{\beta}^{\prime},
$$

where $\boldsymbol{\alpha}$ and $\boldsymbol{\beta}$ are $n \times r$ matrices of rank $r$. Then (20) can be reparameterized as a VEqCM:

$$
\Delta \mathbf{w}_{t}=\boldsymbol{\kappa}+\boldsymbol{\alpha} \boldsymbol{\beta}^{\prime} \mathbf{w}_{t-1}+\boldsymbol{\nu}_{t}
$$

which we write as:

$$
\Delta \mathbf{w}_{t}=\gamma+\boldsymbol{\alpha}\left(\boldsymbol{\beta}^{\prime} \mathbf{w}_{t-1}-\boldsymbol{\mu}\right)+\boldsymbol{\nu}_{t} .
$$

so the cointegration vectors are expressed as deviations about their expectations, and the intercept is the net rate of growth. 
Clements and Hendry (1996b) compare $h$-step forecasts from the correctly-specified model (22) with those from the DVAR, defined by setting $\Delta \mathbf{w}_{T+h}$ equal to the population growth rate $\gamma$ :

$$
\Delta \widetilde{\mathbf{w}}_{T+h}=\gamma
$$

so $h$-step ahead forecasts of the level of the process are:

$$
\widetilde{\mathbf{w}}_{T+j}=\gamma+\widetilde{\mathbf{w}}_{T+j-1}=j \gamma+\mathbf{w}_{T} \text { for } j=1, \ldots, h .
$$

They show that when neither $\boldsymbol{\mu}$ nor $\boldsymbol{\gamma}$ are subject to a structural break, the DVAR yields unbiased forecasts as well as the VEqCM. Moreover, when $\boldsymbol{\mu}$ changes before the forecast origin, the forecasts from the DVAR remain unbiased, but this is not true of VEqCM forecasts.

\section{2 $\mathrm{M}_{5}$}

Consider the cointegrated vector process in (20) for a sequence of 1 -step forecasts, from $T, T+1, \ldots$ when, at $T, \mu$ undergoes a step-change to $\mu^{*}$. In the absence of the break:

$$
\mathbf{w}_{T+1}=\boldsymbol{\kappa}+\boldsymbol{\Gamma} \mathbf{w}_{T}+\boldsymbol{\nu}_{T+1}=\mathbf{w}_{T}+\boldsymbol{\gamma}+\boldsymbol{\alpha}\left(\boldsymbol{\beta}^{\prime} \mathbf{w}_{T}-\boldsymbol{\mu}\right)+\boldsymbol{\nu}_{T+1}
$$

but otherwise:

$$
\mathbf{w}_{T+1}^{*}=\mathbf{w}_{T}+\boldsymbol{\gamma}+\boldsymbol{\alpha}\left(\boldsymbol{\beta}^{\prime} \mathbf{w}_{T}-\boldsymbol{\mu}-\boldsymbol{\delta}_{\mu}\right)+\boldsymbol{\nu}_{T+1}=\mathbf{w}_{T+1}-\boldsymbol{\alpha} \boldsymbol{\delta}_{\mu}
$$

where:

$$
\delta_{\mu}=\mu^{*}-\mu
$$

Recall that the DVAR forecast is defined by:

$$
\widetilde{\mathbf{w}}_{T+1}=\mathbf{w}_{T}+\gamma
$$

so the 1-step ahead DVAR forecast error is:

$$
\mathrm{E}\left[\mathbf{w}_{T+1}^{*}-\widetilde{\mathbf{w}}_{T+1}\right]=-\boldsymbol{\alpha} \boldsymbol{\delta}_{\mu}
$$

since $\mathrm{E}\left[\boldsymbol{\beta}^{\prime} \mathbf{w}_{T}-\boldsymbol{\mu}\right]=\mathbf{0}$. Thus the DVAR forecasts are biased.

Moving the forecast origin forward one period to $T+1$ :

$$
\widetilde{\mathbf{w}}_{T+2}=\mathbf{w}_{T+1}^{*}+\gamma
$$

and the actual value of the process is:

$$
\mathbf{w}_{T+2}^{*}=\mathbf{w}_{T+1}^{*}+\boldsymbol{\gamma}+\boldsymbol{\alpha}\left(\boldsymbol{\beta}^{\prime} \mathbf{w}_{T+1}^{*}-\boldsymbol{\mu}-\boldsymbol{\delta}_{\mu}\right)+\boldsymbol{\nu}_{T+2} .
$$

Thus:

$$
\mathrm{E}\left[\mathbf{w}_{T+2}^{*}-\widetilde{\mathbf{w}}_{T+2}\right]=\boldsymbol{\alpha} \mathrm{E}\left[\boldsymbol{\beta}^{\prime} \mathbf{w}_{T+1}^{*}-\boldsymbol{\mu}-\boldsymbol{\delta}_{\mu}\right]
$$

Substituting for $\mathbf{w}_{T+1}^{*}$ from (25) yields:

$$
\mathrm{E}\left[\mathbf{w}_{T+2}^{*}-\widetilde{\mathbf{w}}_{T+2}\right]=-\boldsymbol{\alpha}\left(\mathbf{I}_{n}+\boldsymbol{\beta}^{\prime} \boldsymbol{\alpha}\right) \boldsymbol{\delta}_{\mu}
$$

For an origin of $T+2$, the 1-step forecast error from the DVAR is:

$$
\mathrm{E}\left[\mathbf{w}_{T+3}^{*}-\widetilde{\mathbf{w}}_{T+3}\right]=\boldsymbol{\alpha}\left(\boldsymbol{\beta}^{\prime} \mathbf{w}_{T+2}^{*}-\boldsymbol{\mu}-\boldsymbol{\delta}_{\mu}\right)=-\boldsymbol{\alpha}\left(\mathbf{I}_{n}+\boldsymbol{\beta}^{\prime} \boldsymbol{\alpha}\right)^{2} \boldsymbol{\delta}_{\mu}
$$


where the solution is again obtained by substituting for $\mathbf{w}_{T+2}^{*}$ in terms of $\mathbf{w}_{T+1}^{*}$, and then for $\mathbf{w}_{T+1}^{*}$ in terms of $\mathbf{w}_{T}$.

More generally, for a 1-step forecast with origin $T+j$ :

$$
\mathrm{E}\left[\mathbf{w}_{T+j+1}^{*}-\widetilde{\mathbf{w}}_{T+j+1}\right]=-\boldsymbol{\alpha}\left(\boldsymbol{\beta}^{\prime} \mathbf{w}_{T+j+1}^{*}-\boldsymbol{\mu}-\boldsymbol{\delta}_{\mu}\right)=-\boldsymbol{\alpha}\left(\mathbf{I}_{n}+\boldsymbol{\beta}^{\prime} \boldsymbol{\alpha}\right)^{j} \boldsymbol{\delta}_{\mu}
$$

where $\left(\mathbf{I}_{n}+\boldsymbol{\beta}^{\prime} \boldsymbol{\alpha}\right)^{p} \rightarrow \mathbf{0}$ as $p \rightarrow \infty$, so that for forecast origins sufficiently long after the break has occurred, the DVAR forecasts are unbiased for changes in $\boldsymbol{\mu}$.

Forecasts from the VEqCM with origin $T$ are given by:

$$
\widehat{\mathbf{w}}_{T+1}=\mathbf{w}_{T}+\boldsymbol{\gamma}+\boldsymbol{\alpha}\left(\boldsymbol{\beta}^{\prime} \mathbf{w}_{T}-\boldsymbol{\mu}\right)
$$

so from (25) the 1-step ahead VEqCM forecast error is:

$$
\mathrm{E}\left[\mathbf{w}_{T+1}^{*}-\widehat{\mathbf{w}}_{T+1}\right]=-\boldsymbol{\alpha} \boldsymbol{\delta}_{\mu}
$$

the same as for the DVAR. This is because the origin $T$ is before the break has occurred (between $T$ and $T+1)$.

The VEqCM forecast from origin $T+1$ is given by:

$$
\widehat{\mathbf{w}}_{T+2}=\mathbf{w}_{T+1}^{*}+\boldsymbol{\gamma}+\boldsymbol{\alpha}\left(\boldsymbol{\beta}^{\prime} \mathbf{w}_{T+1}^{*}-\boldsymbol{\mu}\right)
$$

while:

$$
\mathbf{w}_{T+2}^{*}=\mathbf{w}_{T+1}^{*}+\boldsymbol{\gamma}+\boldsymbol{\alpha}\left(\boldsymbol{\beta}^{\prime} \mathbf{w}_{T+1}^{*}-\boldsymbol{\mu}-\boldsymbol{\delta}_{\mu}\right)+\boldsymbol{\nu}_{T+2} .
$$

thus the 1-step ahead VEqCM forecast error is:

$$
\mathrm{E}\left[\mathbf{w}_{T+2}^{*}-\widehat{\mathbf{w}}_{T+2}\right]=-\boldsymbol{\alpha} \boldsymbol{\delta}_{\mu}
$$

and in general, for 1-step forecasts:

$$
\mathrm{E}\left[\mathbf{w}_{T+j+1}^{*}-\widehat{\mathbf{w}}_{T+j+1}\right]=-\boldsymbol{\alpha} \boldsymbol{\delta}_{\mu}
$$

independently of the origin $(T+j)$. That is, the VEqCM forecast bias is unaffected by the period of disequilibrium adjustment. 